\title{
Aldol Condensation Versus Superbase-Catalyzed Addition of Ketones to Acetylenes: A Quantum-Chemical and Experimental Study
}

Vladimir B. Orel ${ }^{\dagger}{ }^{\ddagger}$ Nadezhda M. Vitkovskaya, ${ }^{\dagger}$ Alexander S. Bobkov,${ }^{\dagger}$ Nadezhda V. Semenova, ${ }^{\ddagger}$ Elena Yu. Schmidt, ${ }^{\star}$ and Boris A. Trofimov

${ }^{\dagger}$ Laboratory of Quantum-Chemical Modeling of Molecular Systems, Irkutsk State University, 1 K. Marx St., 664003 Irkutsk, Russian Federation

$¥$ A.E. Favorsky Irkutsk Institute of Chemistry, Siberian Branch of the Russian Academy of Sciences, 1 Favorsky St., 664033 Irkutsk, Russian Federation

\section{Supplementary Material}

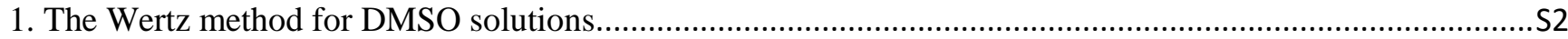

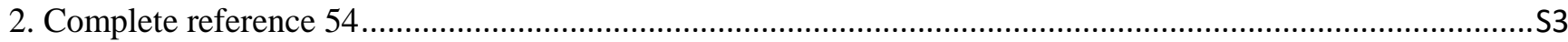

3. Modeling the kinetic curves of the interaction of acetone with acetylene in $\mathrm{KOH} / \mathrm{DMSO}$ superbase. S4

4. Total energies calculated at the B2PLYPD/6-311+G**//B3LYP/6-31+G* level ( $E$, a.u.) and Cartesian coordinates $(\AA)$ of all stationary points: 


\section{The Wertz method for DMSO solutions}

The Wertz approach ${ }^{1}$ suggests that all solutes lose the same fraction of entropy when being dissolved in water. Abraham ${ }^{2}$ has shown that this also holds for other solvents. Wertz also argues that the same amount of entropy is lost when ions are solvated. This allows one to introduce appropriate corrections for an arbitrary solvent.

The Wertz method for DMSO solutions is composed of several steps. In the first step, a solute is treated as an ideal gas and compressed from latm to a hypothetical ideal gas state with the concentration equal to that of the liquid state $(\mathrm{d}=1.100 \mathrm{~g} / \mathrm{ml}, 298 \mathrm{~K}, 14.08 \mathrm{M})$. This entropy change can be estimated as

$$
\Delta S_{1}=-R \ln \frac{P_{2}}{P_{1}}=-R \ln (22.4 \cdot 14.08)=-47.85 \quad \mathrm{~J} \mathrm{~mol}^{-1} \mathrm{~K}^{-1} .
$$

The next step is the conversion of this hypothetical state to the final liquid state. The fraction of entropy lost in the second step is defined as a coefficient, $\alpha$.

$$
\begin{aligned}
& \alpha=\frac{\Delta S_{2}}{S_{g}}=\frac{S_{g}-S_{l}^{0}}{S_{g}}=\frac{S_{g}^{0}+\Delta S_{1}-S_{l}^{0}}{S_{g}}, \\
& \Delta S_{2}=\alpha S_{g}=\alpha S_{g}^{0}+\alpha \Delta S_{1}
\end{aligned}
$$

The entropy of liquid DMSO $S_{l}^{0}=188.78 \mathrm{~J} \mathrm{~mol}^{-1} \mathrm{~K}^{-1}$ (standard state). ${ }^{3}$ The entropy of vaporization can be estimated from the normal heat of vaporization $(52.9 \mathrm{~kJ} / \mathrm{mol})^{4}$ and boiling temperature $(462 \mathrm{~K}$ at $1 \mathrm{~atm})$ as $\Delta S_{\text {vap }}^{0}=114.2 \mathrm{~J} \mathrm{~mol}^{-1} \mathrm{~K}^{-1}$, therefore, $S_{g}^{0}=303.0 \mathrm{~J} \mathrm{~mol}^{-1} \mathrm{~K}^{-1}$ and $\alpha=$ 0.26 .

The entropy change from the gas state of any given molecule $\mathbf{M}$ in the standard state to its $1 \mathrm{M}$ state in DMSO is composed of three steps. The first step is the compression of ideal $\mathbf{M}$ gas in the standard state to a hypothetical ideal gas state with the concentration equal to that of the solvent liquid state $(14.08 \mathrm{M})$ that gives the same $\Delta S_{1}=-47.85 \mathrm{~J} \mathrm{~mol}^{-1} \mathrm{~K}^{-1}$. Conversion of the hypothetical ideal gas state to a hypothetical liquid state brings $\Delta S_{2}=\alpha S_{g}=\alpha S_{g}^{0}+\alpha \Delta S_{1}$. The fraction of entropy loss in this step is assumed to be equal to $\alpha$. Finally, expansion of the hypothetical liquid state to the $1 \mathrm{M}$ state in DMSO results in $\Delta S_{3}=R \ln (14.08)=21.99 \mathrm{~J} \mathrm{~mol}^{-1} \mathrm{~K}^{-1}$.

The calculated gas phase entropy of $\mathbf{M}$ in the standard state is then converted to the corresponding entropy in its $1 \mathrm{M}$ state in DMSO according to the following equation:

$$
S_{l}^{0}=S_{g}^{0}+\Delta S_{1}-\Delta S_{2}+\Delta S_{3}=0.74 \times S_{g}^{0}-13.41 \mathrm{~J} \mathrm{~mol}^{-1} \mathrm{~K}^{-1} \text {, }
$$

For a bimolecular reaction it results in changing

$$
T \Delta S_{l}=0.74 \times T \Delta S_{g}-0.95 \mathrm{kcal} / \mathrm{mol} \text {. }
$$

\section{References:}

(1) Wertz, D. H. Relationship between the Gas-Phase Entropies of Molecules and Their Entropies of Solvation in Water and 1-Octanol. J. Am. Chem. Soc. 1980, 102 (16), 5316-5322. https://doi.org/10.1021/ja00536a033.

(2) Abraham, M. H. Relationship between Solution Entropies and Gas Phase Entropies of Nonelectrolytes. J. Am. Chem. Soc. 1981, 103 (22), 6742-6744.

https://doi.org/10.1021/ja00412a036.

(3) Clever, H. L.; Westrum, E. F. Dimethyl Sulfoxide and Dimethyl Sulfone. Heat Capacities, Enthalpies of Fusion, and Thermodynamic Properties. J. Phys. Chem. 1970, 74 (6), 1309-1317. https://doi.org/10.1021/j100701a027.

(4) Douglas, T. B. Vapor Pressure of Methyl Sulfoxide from 20 to $50^{\circ}$. Calculation of the Heat of Vaporization. J. Am. Chem. Soc. 1948, 70 (6), 2001-2002. https://doi.org/10.1021/ja01186a005. 


\section{Complete reference 54}

M.J. Frisch, G.W. Trucks, H.B. Schlegel, G.E. Scuseria, M.A. Robb, J.R. Cheeseman, G. Scalmani, V. Barone, B. Mennucci, G.A. Petersson, H. Nakatsuji, M. Caricato, X. Li, H.P. Hratchian, A.F. Izmaylov, J. Bloino, G. Zheng, J.L. Sonnenberg, M. Hada, M. Ehara, K. Toyota, R. Fukuda, J. Hasegawa, M. Ishida, T. Nakajima, Y. Honda, O. Kitao, H. Nakai, T. Vreven, J.A. Montgomery, Jr., J.E. Peralta, F. Ogliaro, M. Bearpark, J.J. Heyd, E. Brothers, K. N. Kudin, V.N. Staroverov, T. Keith, R. Kobayashi, J. Normand, K. Raghavachari, A. Rendell, J.C. Burant, S.S. Iyengar, J. Tomasi, M. Cossi, N. Rega, J.M. Millam, M. Klene, J.E. Knox, J.B. Cross, V. Bakken, C. Adamo, J. Jaramillo, R. Gomperts, R.E. Stratmann, O. Yazyev, A.J. Austin, R. Cammi, C. Pomelli, J.W. Ochterski, R.L. Martin, K. Morokuma, V.G. Zakrzewski, G.A. Voth, P. Salvador, J.J. Dannenberg, S. Dapprich, A.D.

Daniels, Ö. Farkas, J.B. Foresman, J.V. Ortiz, J. Cioslowski, and D.J. Fox, Gaussian 09, Revision C.01, Inc., Wallingford CT, 2010. 
3. Modeling the kinetic curves of the interaction of acetone with acetylene in KOH/DMSO superbase

The kinetic curves of the interaction of acetone with acetylene in KOH/DMSO superbase were modeled to the Arrhenius equation $\mathrm{k}(\mathrm{T})=\mathrm{k}_{\mathrm{B}} \mathrm{T} / \mathrm{h} \times \exp \left(-\Delta G^{\ddagger} / \mathrm{RT}\right)$, using the KINET program ${ }^{1}$, which allowed for the evaluation of the reaction rate constants at $\mathrm{T}=353 \mathrm{~K}$ :

\begin{tabular}{|c|c|c|c|c|}
\hline No & Reaction & $\Delta G^{\ddagger}, \mathrm{kcal} / \mathrm{mol}$ & $\mathrm{k}(\mathrm{T})$ & $\begin{array}{c}\text { Reaction rate by law of } \\
\text { mass action }\end{array}$ \\
\hline 1 & $\mathbf{1}+\mathrm{KOH} \cdot \mathrm{DMSO} \rightarrow \mathbf{2}$ & 8.0 & $8.25 \times 10^{7}$ & $\mathrm{R} 1=\mathrm{k} 1 \times \mathrm{C}_{1} \times \mathrm{C}_{\mathrm{KOH}}$ \\
\hline 2 & $\mathbf{2} \rightarrow \mathbf{1}+\mathrm{KOH} \cdot \mathrm{DMSO}$ & 10.8 & $1.53 \times 10^{6}$ & $\mathrm{R} 2=\mathrm{k} 2 \times \mathrm{C}_{2}$ \\
\hline 3 & $\mathbf{2}+\mathbf{1 3} \rightarrow \mathbf{1 8}+\mathrm{KOH} \cdot \mathrm{DMSO}$ & 22.8 & $5.72 \times 10^{-2}$ & $\mathrm{R} 3=\mathrm{k} 3 \times \mathrm{C}_{2} \times \mathrm{C}_{13}$ \\
\hline 4 & $\mathbf{1 8}+\mathrm{KOH} \cdot \mathrm{DMSO} \rightarrow \mathbf{2}+\mathbf{1 3}$ & 35.0 & $1.61 \times 10^{-9}$ & $\mathrm{R} 4=\mathrm{k} 4 \times \mathrm{C}_{18} \times \mathrm{C}_{\mathrm{KOH}}$ \\
\hline 5 & $\mathbf{1}+\mathbf{2} \rightarrow \mathbf{6}+\mathrm{KOH} \cdot \mathrm{DMSO} \cdot \mathrm{H}_{2} \mathrm{O}$ & 15.9 & $1.07 \times 10^{3}$ & $\mathrm{R} 5=\mathrm{k} 5 \times \mathrm{C}_{1} \times \mathrm{C}_{2}$ \\
\hline 6 & $\mathbf{6}+\mathrm{KOH} \cdot \mathrm{DMSO} \cdot \mathrm{H}_{2} \mathrm{O} \rightarrow \mathbf{1}+\mathbf{2}$ & 17.7 & $8.20 \times 10^{1}$ & $\mathrm{R} 6=\mathrm{k} 6 \times \mathrm{C}_{6} \times \mathrm{C}_{\mathrm{KOH}}$ \\
\hline 7 & $6+2 \rightarrow 7$ & 13.6 & $3.26 \times 10^{4}$ & $\mathrm{R} 7=\mathrm{k} 7 \times \mathrm{C}_{6} \times \mathrm{C}_{2}$ \\
\hline 8 & $7 \rightarrow 6+2$ & 18.1 & $5.34 \times 10^{1}$ & $\mathrm{R} 8=\mathrm{k} 8 \times \mathrm{C}_{7}$ \\
\hline 9 & $7 \rightarrow 9 a$ & 17.5 & $1.26 \times 10^{2}$ & $\mathrm{R} 9=\mathrm{k} 9 \times \mathrm{C}_{7}$ \\
\hline 10 & $\mathbf{9 a} \rightarrow 7$ & 22.7 & $7.61 \times 10^{-2}$ & $\mathrm{R} 10=\mathrm{k} 10 \times \mathrm{C}_{9 \mathrm{a}}$ \\
\hline 11 & $\mathbf{9 a} \rightarrow \mathbf{1 2 a}+\mathrm{KOH} \cdot \mathrm{DMSO} \cdot \mathrm{H}_{2} \mathrm{O}$ & 12.7 & $1.02 \times 10^{5}$ & $\mathrm{R} 11=\mathrm{k} 11 \times \mathrm{C}_{9 \mathrm{a}}$ \\
\hline 12 & $\mathbf{1 2 a}+\mathrm{KOH} \cdot \mathrm{DMSO} \cdot \mathrm{H}_{2} \mathrm{O} \rightarrow \mathbf{9 a}$ & 20.7 & 1.14 & $\mathrm{R} 12=\mathrm{k} 12 \times \mathrm{C}_{12 \mathrm{a}} \times \mathrm{C}_{\mathrm{KOH}}$ \\
\hline 13 & $13+\mathrm{KOH} \cdot \mathrm{DMSO} \rightarrow \mathbf{1 4}$ & 1.1 & $1.54 \times 10^{12}$ & $\mathrm{R} 13=\mathrm{k} 13 \times \mathrm{C}_{13} \times \mathrm{C}_{\mathrm{KOH}}$ \\
\hline 14 & $\mathbf{1 4} \rightarrow \mathbf{1 3}+\mathrm{KOH} \cdot \mathrm{DMSO}$ & 1.9 & $4.91 \times 10^{11}$ & $\mathrm{R} 14=\mathrm{k} 14 \times \mathrm{C}_{14}$ \\
\hline 15 & $14+1 \rightarrow 15$ & 12.4 & $1.56 \times 10^{5}$ & $\mathrm{R} 15=\mathrm{k} 15 \times \mathrm{C}_{14} \times \mathrm{C}_{1}$ \\
\hline 16 & $15 \rightarrow 14+1$ & 19.4 & 7.27 & $\mathrm{R} 16=\mathrm{k} 16 \times \mathrm{C}_{15}$ \\
\hline 17 & $\mathbf{1 5}+\mathrm{H}_{2} \mathrm{O} \rightarrow \mathbf{1 6}+\mathrm{KOH} \cdot \mathrm{DMSO} \cdot \mathrm{H}_{2} \mathrm{O}$ & $2.8^{*}$ & $1.36 \times 10^{11}$ & $\mathrm{R} 17=\mathrm{k} 17 \times \mathrm{C}_{15} \times \mathrm{C}_{\mathrm{HOH}}$ \\
\hline 18 & $\mathbf{1 6}+\mathrm{KOH} \cdot \mathrm{DMSO} \cdot \mathrm{H}_{2} \mathrm{O} \rightarrow \mathbf{1 5}+\mathrm{H}_{2} \mathrm{O}$ & $0.0 *$ & $1.36 \times 10^{12}$ & $\mathrm{R} 18=\mathrm{k} 18 \times \mathrm{C}_{16} \times \mathrm{C}_{\mathrm{KOH}}$ \\
\hline 19 & $16+15 \rightarrow 17$ & 22.9 & $4.96 \times 10^{-2}$ & $\mathrm{R} 19=\mathrm{k} 19 \times \mathrm{C}_{16} \times \mathrm{C}_{15}$ \\
\hline 20 & $17 \rightarrow 16+15$ & 32.4 & $6.56 \times 10^{-8}$ & $\mathrm{R} 20=\mathrm{k} 20 \times \mathrm{C}_{17}$ \\
\hline
\end{tabular}

* -The transition state of the protonation of alcohol anion molecule by a water molecule has not been localized. Upon proton transfer from the water molecule to the $O$-anion center of 2-methylbut-3-yn-2-olate, the energy of the system monotonously increases, and the methyl-3-butyn-2-ol complex is eventually formed.

\section{Input concentrations:}

The initial concentration of the starting reagents was: (a) methyl-3-butyn-2-ol C16 $=0.4 \mathrm{~mol} / \mathrm{L}$ and acetylene $\mathrm{C}_{13}=0.4 \mathrm{~mol} / \mathrm{L} ;(\mathbf{b})$ acetone $\mathrm{C}_{1}=2.0 \mathrm{~mol} / \mathrm{L}$ and acetylene $\mathrm{C}_{13}=0.5 \mathrm{~mol} / \mathrm{L}$. In both (a) and (b) cases, the initial concentration of water $\mathrm{C}_{\mathbf{H O H}}=0.1 \mathrm{~mol} / \mathrm{L}$ in DMSO was calculated based on its $0.2 \%$ content in DMSO; the concentration of potassium hydroxide $\mathrm{CKон}_{\mathbf{K}}=0.037 \mathrm{~mol} / \mathrm{L}$ was taken constant and equivalent to its solubility in DMSO.

To determine the concentration of acetylene, its volume dissolved in DMSO at ambient temperature was calculated from the experimental data $\left(\mathrm{V}_{\text {reactor }}=0.25 \mathrm{~L} ; \mathrm{P}_{\text {final }}=2 \mathrm{~atm} ;(\mathbf{a}) \mathrm{V}_{\text {DMSO }}=\right.$ $0.06 \mathrm{~L}, \mathrm{P}_{\text {initial }}=12 \mathrm{~atm}$; (b) $\left.\mathrm{V}_{\mathrm{DMSO}}=0.05 \mathrm{~L} ; \mathrm{P}_{\text {initial }}=14 \mathrm{~atm}\right)$ according to Boyle's law $(\mathrm{PV}=$ const. $)$ as 
$\mathrm{V}_{\mathrm{HCCH}, 298}=\left(\mathrm{V}_{\text {reactor }}-\mathrm{V}_{\text {DMSO }}\right) \times\left(\mathrm{P}_{\text {initial }}-\mathrm{P}_{\text {final }}\right) / 1 \mathrm{~atm}$.

This volume was then recalculated taking the synthesis temperature $(\mathrm{T}=353 \mathrm{~K})$ into account as $\lg \mathrm{V}_{\mathrm{HCCH}, 298}=\lg \mathrm{V}_{\mathrm{HCCH}, \mathrm{T}}+0.0113(\mathrm{~T}-298){ }^{2}$

\section{References:}

(1) A.V. Abramenkov, Kinet for Windows, version 20.8. http://www.chem.msu.su/rus/teaching/KINET2012

(2) McKinnis, A. C. Solubility of Acetylene in Donor Solvents. Ind. Eng. Chem. 1955, 47 (4), 850-853. https://doi.org/10.1021/ie50544a053 
4. Total energies calculated at the B2PLYPD/6-311+G**//B3LYP/6-31+G* level (E, a.u.) and Cartesian coordinates $(\AA)$ of all stationary points:

Figure 1

\begin{tabular}{|c|c|c|c|c|}
\hline 6 & -1.289961000 & -0.613026000 & -0.002373000 & \\
\hline 6 & 0.000012000 & 0.177620000 & 0.000381000 & \\
\hline 6 & 1.289976000 & -0.613010000 & 0.002241000 & \\
\hline 8 & -0.000014000 & 1.404329000 & -0.000100000 & \\
\hline 1 & 1.276798000 & -1.372321000 & 0.793235000 & \\
\hline 1 & 2.148238000 & 0.049627000 & 0.135200000 & \\
\hline 1 & 1.390241000 & -1.149371000 & -0.950501000 & \\
\hline 1 & -1.275926000 & -1.373220000 & -0.792486000 & \\
\hline 1 & -2.148081000 & 0.049475000 & -0.136969000 & 1 \\
\hline 1 & -1.391324000 & -1.148329000 & 0.950826000 & $=-193.0438118$ a.u. \\
\hline 6 & 4.282037000 & 1.452819000 & 0.128289000 & \multirow{23}{*}{$\begin{array}{c}\mathbf{2} \\
\mathrm{E}(\mathrm{B} 2 \mathrm{PLYPD})=-1421.7014798 \text { a.u. }\end{array}$} \\
\hline 6 & 3.653239000 & 0.235316000 & 0.096445000 & \\
\hline 6 & 4.456403000 & -1.034848000 & 0.358560000 & \\
\hline 8 & 2.377244000 & 0.043859000 & -0.145300000 & \\
\hline 19 & 0.377878000 & -1.643353000 & -0.217674000 & \\
\hline 8 & -1.547661000 & 0.272374000 & -0.085029000 & \\
\hline 16 & -3.041353000 & 0.663214000 & 0.030137000 & \\
\hline 6 & -3.936262000 & -0.465649000 & -1.086788000 & \\
\hline 6 & -3.612994000 & -0.029425000 & 1.616098000 & \\
\hline 8 & 0.636261000 & 2.100162000 & -0.483067000 & \\
\hline 1 & 1.350618000 & 1.408696000 & -0.360292000 & \\
\hline 1 & -5.010964000 & -0.303052000 & -0.965119000 & \\
\hline 1 & -3.636077000 & -0.213920000 & -2.106364000 & \\
\hline 1 & -3.667871000 & -1.499310000 & -0.851598000 & \\
\hline 1 & -4.692803000 & 0.123666000 & 1.698718000 & \\
\hline 1 & -3.363091000 & -1.093045000 & 1.659292000 & \\
\hline 1 & -3.098951000 & 0.517489000 & 2.409511000 & \\
\hline 1 & 5.345100000 & 1.532961000 & 0.338612000 & \\
\hline 1 & 3.732812000 & 2.374914000 & -0.054713000 & \\
\hline 1 & -0.197990000 & 1.602574000 & -0.353947000 & \\
\hline 1 & 4.040856000 & -1.572685000 & 1.221934000 & \\
\hline 1 & 4.383004000 & -1.712278000 & -0.503374000 & \\
\hline 1 & 5.516044000 & -0.834169000 & 0.552514000 & \\
\hline 6 & -3.364694000 & -3.205477000 & -0.869150000 & \multirow{21}{*}{$\mathrm{E}(\mathrm{B} 2 \mathrm{PLYPD})=-1614.770061$ a.u. } \\
\hline 6 & -3.020483000 & -2.156808000 & 0.184568000 & \\
\hline 8 & -1.758808000 & -1.802189000 & 0.238790000 & \\
\hline 6 & -4.011612000 & -1.657591000 & 0.990459000 & \\
\hline 6 & -1.519857000 & 3.311178000 & -0.201928000 & \\
\hline 6 & -2.495726000 & 2.736728000 & -1.201489000 & \\
\hline 6 & -1.398102000 & 4.815321000 & -0.138695000 & \\
\hline 8 & -0.851316000 & 2.586178000 & 0.531185000 & \\
\hline 19 & -0.150180000 & 0.022613000 & 1.270287000 & \\
\hline 8 & 2.028475000 & -0.783457000 & -0.180738000 & \\
\hline 16 & 3.551562000 & -0.692840000 & -0.443052000 & \\
\hline 6 & 4.336642000 & -0.676546000 & 1.201688000 & \\
\hline 6 & 3.870718000 & 1.041731000 & -0.903269000 & \\
\hline 1 & -3.518344000 & 3.002102000 & -0.902866000 & \\
\hline 1 & -2.405045000 & 1.649520000 & -1.251763000 & \\
\hline 1 & -2.332786000 & 3.173767000 & -2.193746000 & \\
\hline 1 & -5.041309000 & -1.991233000 & 0.894238000 & \\
\hline 1 & -3.790984000 & -0.909078000 & 1.750367000 & \\
\hline 1 & -2.779329000 & -4.119357000 & -0.698646000 & \\
\hline 1 & -4.427885000 & -3.470284000 & -0.870374000 & \\
\hline 1 & -3.096934000 & -2.839125000 & -1.869711000 & \\
\hline
\end{tabular}


$-1.070358000$

$1-2.388057000$ 5.280100000

$-0.062309000$

$-0.775769000$

5.117472000

0.706523000

$1 \quad 5.409360000$

$-0.502077000$

1.079698000

3.879831000

0.105546000

1.814240000

14.168894000

4.950629000

$-1.659521000$

1.647402000

13.392780000

1.190565000

$-0.991188000$

3.443971000

1.210681000

$-1.870670000$

0.968046000

1.702845000

$-0.144000000$

0.266443000

$-2.146513000$

$-0.993279000$

$-0.541026000$

$-2.762492000$

$-1.291573000$

$-2.817517000$

$-2.476932000$

$-0.774597000$

$-2.435613000$

1.400761000

0.421784000

$-1.220715000$

1.340547000

$-0.513808000$

$-3.394322000$

0.421412000

$-0.895620000$

$-0.855234000$

$-3.068218000$

$-1.193436000$

0.481031000

$-3.395725000$

$-0.556449000$

1.833501000

$-4.197624000$

$-2.069454000$

$-0.073658000$

$-1.871757000$

$-1.603179000$

0.301591000

$19 \quad 0.279739000$

$-0.935295000$

$-1.058131000$

2.502747000

0.286452000

0.001546000

0.242997000

0.196767000

$-1.063161000 \quad-0.921617000$

4.641950000

$-0.616531000$

1.780205000

4.313022000

$-0.039982000$

1.839082000

.3609030

0.142348000

2.133684000

$-3.435277000$

$-1.359189000$

2.586200000

$-4.442264000$

0.664897000

$-0.694617000$

$-3.185039000$

$-0.178200000$

$-1.738180000$

$-2.685082000$

3.494515000

$-0.100554000$

$-3.852595000$

2.473025000

0.768914000

$-2.149929000$

2.553030000

1.292586000

$-4.264567000$

$-2.969595000$

$-5.172667000$

$-1.572253000$

0.556649000

$-0.057325000$

$-3.972427000$

$-2.390979000$

$-1.095195000$

5.710494000

$-1.213694000$

$-0.743577000$

4.081851000

$-1.984679000$

$-0.740972000$

4.482356000

$-0.711127000$

$-1.943216000$

5.387937000

$-0.763379000$

1.919271000

3.922562000

0.031255000

2.568137000

3.783258000

$-1.573043000$

1.605787000

1.942946000

1.772989000

0.091385000

0.075905000

$0.990530000 \quad 2.707623000$

$-0.239652000$

$3.410300000 \quad 2.355615000$

$-1.172835000$

$-0.055149000$

0.324359000

1.575566000

1.646402000

2.064630000

0.576690000

3.323674000

0.448122000

$-0.961729000$

3.000308000

$-1.086083000$

0.057558000

$-1.469300000$

$3.631006000-2.031696000$

0.772100000

0.376689000

$-1.106964000$

1.483441000

$-0.355800000$

0.452378000

$-0.389554000$

$-0.063659000$

$-0.173686000$

$-0.658266000$

$-3.862978000$

1.629753000

$-0.667346000$

-4.129288000
-5.032962000

$-0.599756000$

0.673484000

4.048654000

$-0.920288000$

$-1.753842000$

2.373646000

$-0.374719000$

$-2.020732000$

2.717922000

$-2.094940000$

$-1.795648000$

4.407471000

0.490269000

0.414778000

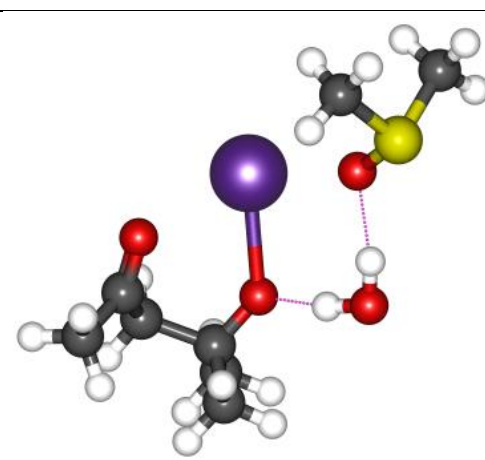

3

$\mathrm{E}(\mathrm{B} 2 \mathrm{PLYPD})=-1614.790238$ a.u. 
0.472009000

1.653970000

4.228503000

2.943343000

$-0.733386000$

3.869999000

1.645021000

$-1.867140000$

2.741294000

3.032084000

$-1.711139000$

3.294029000

$-3.029692000$

4.701110000

$-1.942341000$

0.463565000

3.506166000

$-1.953312000$

0.538143000

$-5.159939000$

1.831988000

1.859538000

$-3.930719000$

2.030901000

$-0.972406000$

$-3.435970000$

2.054648000

0.330333000

$-6.047019000$

$-0.357905000$

$-1.396510000$

0.342457000

$-4.945605000$

$-1.674507000$

0.847361000

$-4.772186000$

$-0.042657000$

$-1.208731000$

$-1.627549000$

$-0.449468000$

$-2.180132000$

1.577547000

$-0.885816000$

0.332995000

$-1.806179000$

$-1.162067000$

$-2.699530000$

3.088248000

$-0.637954000$

$-2.129758000$

1.707778000

$-0.933727000$

1.483817000

$-0.370873000$

$-0.188622000$

$-3.067978000$

0.628389000

$-0.868581000$

$-3.545377000$

$-0.393644000$

0.229274000

$-4.190928000$

0.322880000

1.423150000

$-4.569538000$

$-1.337512000$

$-0.430370000$

$-2.447989000$

$-1.133704000$

0.738425000

0.217162000

3.097093000

$-0.842500000$

0.092164000

$-0.221943000$

$-0.437481000$

0.805258000

5.629941000

$-0.800598000$

$-0.118228000$

4.966204000

1.706998000

1.107974000

1.955423000

$-3.458654000$

.961611000

2.130561000

$-4.577816000$

$-0.419402000$

1.093216000

$-1.321741000$

-3.950873000
-2.545967000

0.052023000

$-1.640101000$

$-2.952279000$

3.535550000

$-1.105920000$

$-3.631162000$

3.040299000

0.439545000

$-1.973478000$

3.725967000

$-4.898224000$

$-2.088710000$

0.375279000

$-5.451411000$

$-0.789473000$

0.297351000

$-0.783422000$

$-4.124035000$

$-1.859542000$

$-1.285154000$

6.670609000

$-0.602479000$

5.398294000

$-0.392319000$

0.532837000

1.793050000

0.783161000

6.015820000

$-1.876048000$

$-0.380729000$

4.326701000

1.867320000

$-0.758520000$

0.935258000

$-1.321990000$

$-1.571538000$

1.938762000

$-3.277326000$

$-0.904241000$

$-1.088265000$

$-2.548857000$

0.008791000 


\begin{tabular}{|c|c|c|c|}
\hline 6 & 3.227724000 & 2.859478000 & -0.455380000 \\
\hline 6 & 2.605372000 & 1.468560000 & -0.541173000 \\
\hline 8 & 1.511147000 & 1.356089000 & -1.155177000 \\
\hline 6 & 3.261608000 & 0.400193000 & 0.165657000 \\
\hline 6 & 3.102871000 & -1.084033000 & -0.231601000 \\
\hline 6 & 3.098551000 & -1.326108000 & -1.745223000 \\
\hline 6 & 4.239800000 & -1.890718000 & 0.421787000 \\
\hline 8 & 1.836136000 & -1.625165000 & 0.253844000 \\
\hline 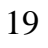 & -0.435882000 & 0.228428000 & 5405000 \\
\hline 8 & -3.067553000 & -0.015397000 & 0.219285000 \\
\hline 16 & -4.571903000 & -0.322338000 & 0.363656000 \\
\hline 6 & -5.035538000 & -1.282668000 & -1.116937000 \\
\hline 6 & -5.443587000 & 1.224344000 & -0.056759000 \\
\hline 1 & 4.053121000 & -1.010523000 & -2.182612000 \\
\hline 1 & 2.293834000 & -0.762825000 & -2.224476000 \\
\hline 1 & 2.959715000 & -2.393744000 & 9070000 \\
\hline 1 & 4.288534000 & 0.647960000 & 0.446202000 \\
\hline 1 & 2.532888000 & 0.440048000 & 847000 \\
\hline 1 & 3.994550000 & 2.936145000 & 667000 \\
\hline 1 & 3.697647000 & 3.094942000 & 225000 \\
\hline 1 & 2.450295000 & 3.610 & 3000 \\
\hline 1 & 4.110875000 & 7000 & 4000 \\
\hline 1 & 5.219021000 & -1.5 & 000 \\
\hline 1 & 4.241512000 & -1.748 & 874000 \\
\hline 1 & -6.119009000 & 84000 & 7267000 \\
\hline 1 & -4.713912000 & -0.745460000 & 42000 \\
\hline 1 & -4.527576000 & -2.247148000 & 46880000 \\
\hline 1 & -6.519576000 & 0000 & 318000 \\
\hline 1 & -5.215590000 & 40000 & 4000 \\
\hline 1 & -5.088521000 & & \\
\hline 9 & 2.123713000 & $0.1^{\prime}$ & 000 \\
\hline 8 & 1.666129000 & 0.196670000 & 81000 \\
\hline 1 & 1.743012000 & -1.289 & 520000 \\
\hline 6 & 2.930667000 & 2.787679000 & 31000 \\
\hline 6 & 2.447142 & 1.34 & \\
\hline 8 & 1.245253000 & 1.108 & 850000 \\
\hline 6 & 3.312196000 & 0.419662000 & -0.257382000 \\
\hline 6 & 3.110467000 & 10000 & 967000 \\
\hline 6 & 2.689319000 & -1.723547000 & -1.500610000 \\
\hline 6 & 4.398141000 & -1.748863000 & 0.344020000 \\
\hline 8 & 2.023479000 & 81000 & 64000 \\
\hline 19 & -0.379737000 & 0.048349000 & 3748000 \\
\hline 8 & -3.020523000 & 44000 & 0.410525000 \\
\hline 16 & -4.551508000 & -0.345110000 & 0.361783000 \\
\hline 6 & -4.907445000 & -1.247751000 & -1.183781000 \\
\hline 6 & -5.230764000 & 1.275414000 & -0.131629000 \\
\hline 1 & 3.487424000 & -1.604311000 & -2.242282000 \\
\hline 1 & & & \\
\hline 1 & 2.499249000 & -2.796872000 & -1.368610000 \\
\hline 1 & 4.311485000 & 0.772694000 & -0.005990000 \\
\hline 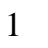 & 2.412220000 & 1.069954000 & 1.540228000 \\
\hline 1 & 3.940246000 & 2.949142000 & -0.587639000 \\
\hline 1 & 2.923735000 & 3.070729000 & -2.043782000 \\
\hline 1 & 2.239272000 & 3.467754000 & -0.466906000 \\
\hline 1 & 4.255530000 & -2.831171000 & 0.444468000 \\
\hline 1 & 5.233159000 & -1.571207000 & -0.344684000 \\
\hline 1 & 4.679500000 & -1.344863000 & 1.325295000 \\
\hline 1 & -5.991242000 & -1.304793000 & -1.320068000 \\
\hline 1 & -4.433663000 & -0.731291000 & -2.023246000 \\
\hline 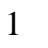 & -4.494501000 & -2.252761000 & -1.071617000 \\
\hline 1 & -6.308705000 & 1.176003000 & -0.288799000 \\
\hline 1 & -5.038626000 & 1.968434000 & 0.690548000 \\
\hline
\end{tabular}
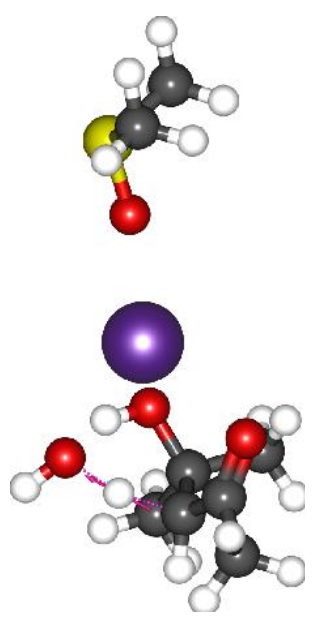

$\mathbf{T S}_{4 \rightarrow 5}$

$\mathrm{E}(\mathrm{B} 2 \mathrm{PLYPD})=-1614.787000$ a.u., $i 1333 \mathrm{~cm}^{-1}$ 


\begin{tabular}{|c|c|c|c|c|}
\hline 1 & -4.731204000 & 1.616018000 & -1.042956000 & \\
\hline 1 & 2.184741000 & 1.482539000 & 3.040142000 & \\
\hline 8 & 1.802160000 & 0.960273000 & 2.317247000 & \\
\hline 1 & 2.183740000 & -0.977705000 & 1.596100000 & \\
\hline 6 & 3.122981000 & 2.862556000 & -0.594956000 & \\
\hline 6 & 2.536286000 & 1.462957000 & -0.761886000 & \\
\hline 8 & 1.362257000 & 1.384533000 & -1.227838000 & \\
\hline 6 & 3.345321000 & 0.362448000 & -0.359729000 & \\
\hline 6 & 3.084006000 & -1.007731000 & -0.526524000 & \\
\hline 6 & 2.193130000 & -1.527206000 & -1.631295000 & \\
\hline 6 & 4.201745000 & -1.953296000 & -0.139121000 & \\
\hline 8 & 1.756274000 & -1.525635000 & 1.012676000 & \\
\hline 19 & -0.289771000 & 0.188100000 & 0.640063000 & \\
\hline 8 & -2.876877000 & -0.002508000 & 0.067571000 & \\
\hline 16 & -4.257031000 & 0.101607000 & -0.612458000 & \\
\hline 6 & -5.322427000 & 1.023979000 & 0.546808000 & \\
\hline 6 & -5.037444000 & -1.536599000 & -0.422125000 & \\
\hline 1 & 2.765846000 & -1.506552000 & -2.571893000 & \\
\hline 1 & 1.307657000 & -0.909170000 & -1.770354000 & \\
\hline 1 & 1.896827000 & -2.562756000 & -1.444393000 & $\rightarrow 6^{\prime}$ \\
\hline 1 & 4.248122000 & 0.617945000 & 0.192551000 & $\mathrm{E}(\mathrm{B} 2 \mathrm{PI}$ \\
\hline 1 & 1.933258000 & -0.422264000 & 2.166827000 & $\mathrm{~cm}^{-1}$ \\
\hline 1 & 4.123339000 & 2.863063000 & -0.151381000 & \\
\hline 1 & 3.175354000 & 3.348532000 & -1.577540000 & \\
\hline 1 & 2.455931000 & 3.470354000 & 0.029032000 & \\
\hline 1 & 3.840739000 & -2.977900000 & -0.006692000 & \\
\hline 1 & 4.950096000 & -1.973207000 & -0.945018000 & \\
\hline 1 & 4.705517000 & -1.631378000 & 0.777304000 & \\
\hline 1 & -6.346286000 & 1.027583000 & 0.161995000 & \\
\hline 1 & -5.276299000 & 0.555053000 & 1.533783000 & \\
\hline 1 & -4.939662000 & 2.046084000 & 0.591577000 & \\
\hline 1 & -6.065546000 & -1.485071000 & -0.791861000 & \\
\hline 1 & -4.460746000 & -2.239777000 & -1.027190000 & \\
\hline 1 & -5.013806000 & -1.830073000 & 0.631113000 & \\
\hline 1 & 2.560883000 & 0.978452000 & 2.506359000 & \\
\hline 8 & 1.882206000 & 0.358814000 & 2.818316000 & \\
\hline 1 & 2.024440000 & -2.412825000 & 1.301070000 & \\
\hline 6 & 2.543077000 & -1.872590000 & -1.960011000 & \\
\hline 6 & 2.863114000 & -0.856142000 & -0.880992000 & \\
\hline 8 & 1.965405000 & -0.148029000 & -0.403945000 & \\
\hline 6 & 4.273372000 & -0.778796000 & -0.473667000 & \\
\hline 6 & 4.795790000 & -0.070424000 & 0.560442000 & \\
\hline 6 & 4.002343000 & 0.790708000 & 1.507239000 & \\
\hline 6 & 6.278148000 & -0.109183000 & 0.819983000 & \\
\hline 8 & -1.115556000 & 3.483300000 & -0.512158000 & \\
\hline 19 & -0.474916000 & 1.025073000 & -0.119693000 & \\
\hline 8 & -3.033813000 & 0.064040000 & 0.090050000 & \\
\hline 16 & -4.055624000 & -1.045235000 & 0.434550000 & \\
\hline 6 & -3.363585000 & -1.933032000 & 1.868689000 & \\
\hline 6 & -3.816893000 & -2.348662000 & -0.817595000 & \\
\hline 1 & 4.514605000 & 0.870989000 & 2.472236000 & ) \\
\hline 1 & 2.986686000 & 0.420932000 & 1.654872000 & \\
\hline 1 & 3.916712000 & 1.808580000 & 1.100284000 & \\
\hline 1 & 4.955351000 & -1.378762000 & -1.073523000 & -1614.762019 a.u. \\
\hline 1 & -2.707824000 & 3.145162000 & -0.502562000 & \\
\hline 1 & 3.120950000 & -1.655809000 & -2.867490000 & \\
\hline 1 & 2.831696000 & -2.879704000 & -1.633366000 & \\
\hline 1 & 1.476650000 & -1.855445000 & -2.195133000 & \\
\hline 1 & 6.685252000 & 0.911167000 & 0.838477000 & \\
\hline 1 & 6.478367000 & -0.538577000 & 1.811218000 & \\
\hline 1 & 6.820910000 & -0.690978000 & 0.069589000 & \\
\hline 1 & -3.993400000 & -2.801226000 & 2.082433000 & \\
\hline
\end{tabular}




\begin{tabular}{lrrrr}
1 & -2.337325000 & -2.238589000 & 1.646932000 \\
1 & -3.383337000 & -1.242801000 & 2.714923000 \\
1 & -4.440151000 & -3.208324000 & -0.555183000 \\
1 & -4.138233000 & -1.935992000 & -1.776455000 \\
1 & -2.760264000 & -2.627530000 & -0.855102000 \\
1 & -3.622222000 & 1.881349000 & -0.278639000 \\
8 & -3.682383000 & 2.837224000 & -0.479330000 \\
1 & -1.012437000 & 4.439373000 & -0.633603000 \\
6 & -2.565236000 & -0.793547000 & -0.105188000 \\
6 & -1.330841000 & 0.079508000 & 0.036552000 \\
6 & -0.045096000 & -0.643859000 & 0.061053000 \\
6 & 1.205648000 & -0.121847000 & -0.003417000 \\
6 & 2.401925000 & -1.037069000 & 0.035844000 \\
8 & -1.441383000 & 1.306726000 & 0.134100000 \\
6 & 1.531619000 & 1.343615000 & -0.114430000 \\
1 & -2.502541000 & -1.404605000 & -1.014430000 \\
1 & -2.634044000 & -1.489581000 & 0.740919000 \\
1 & -3.465068000 & -0.175311000 & -0.139903000 \\
1 & -0.136657000 & -1.727647000 & 0.114291000 \\
1 & 1.792436000 & 1.739012000 & 0.878418000 \\
1 & 2.412288000 & 1.488121000 & -0.751628000 \\
1 & 0.695534000 & 1.931554000 & -0.492488000 \\
1 & 2.123322000 & -2.084348000 & 0.184461000 \\
1 & 3.083450000 & -0.736418000 & 0.843419000 \\
1 & 2.974229000 & -0.955392000 & -0.898343000 \\
\hline & & &
\end{tabular}

Figure 2

\begin{tabular}{|c|c|c|c|c|}
\hline 6 & -1.060767000 & 4.797428000 & 0.884643000 & \\
\hline 6 & -0.602466000 & 3.973448000 & -0.110831000 & \\
\hline 6 & 0.286120000 & 4.544957000 & -1.211494000 & \\
\hline 8 & -0.861112000 & 2.691554000 & -0.217343000 & \\
\hline 19 & -0.386300000 & 0.407625000 & -1.440128000 & \\
\hline 8 & -2.244337000 & -0.999775000 & -0.015382000 & \\
\hline 16 & -2.983727000 & -2.358896000 & -0.056505000 & \\
\hline 6 & -2.991287000 & -2.975103000 & 1.659497000 & \\
\hline 6 & -4.756721000 & -1.958728000 & -0.200406000 & \\
\hline 8 & -2.546306000 & 1.428212000 & 1.494504000 & \\
\hline 1 & -1.935270000 & 1.967483000 & 0.911371000 & \\
\hline 1 & -3.588833000 & -3.890207000 & 1.702858000 & \\
\hline 1 & -1.955138000 & -3.196014000 & 1.925334000 & \\
\hline 1 & -3.401815000 & -2.207791000 & 2.321464000 & (11 \\
\hline 1 & -5.334168000 & -2.885206000 & -0.133590000 & 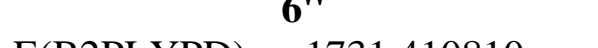 \\
\hline 1 & -5.039267000 & -1.262383000 & 0.593722000 & $\mathrm{E}(\mathrm{B} 2 \mathrm{PLYPD})=-1731.410810$ a.u. \\
\hline 1 & -4.903337000 & -1.500428000 & -1.180935000 & \\
\hline 1 & -2.554390000 & 0.542262000 & 1.076264000 & \\
\hline 1 & 0.477135000 & 5.617013000 & -1.089012000 & \\
\hline 1 & 1.250944000 & 4.019542000 & -1.227828000 & \\
\hline 1 & -0.180100000 & 4.384591000 & -2.193483000 & \\
\hline 1 & -0.801788000 & 5.852548000 & 0.906579000 & \\
\hline 1 & -1.695407000 & 4.416422000 & 1.683068000 & \\
\hline 6 & 3.887730000 & -0.606041000 & 1.145447000 & \\
\hline 6 & 3.897233000 & -1.522358000 & 0.142796000 & \\
\hline 6 & 2.955796000 & -1.607715000 & -0.980466000 & \\
\hline 1 & 4.681944000 & -2.276514000 & 0.146998000 & \\
\hline 6 & 3.259904000 & -2.665740000 & -2.023313000 & \\
\hline 8 & 1.960194000 & -0.879421000 & -1.099990000 & \\
\hline 1 & 3.285223000 & -3.659780000 & -1.558964000 & \\
\hline 1 & 2.504784000 & -2.651154000 & -2.812234000 & \\
\hline 1 & 4.251644000 & -2.494947000 & -2.461062000 & \\
\hline 6 & 2.883336000 & 0.510273000 & 1.276789000 & \\
\hline 1 & 2.728733000 & 1.030862000 & 0.327685000 & \\
\hline
\end{tabular}




\begin{tabular}{|c|c|c|c|}
\hline 1 & 1.904031000 & 0.113029000 & 000 \\
\hline 1 & 3.200916000 & 1.230021000 & 2.037576000 \\
\hline 6 & 4.930678000 & -0.669775000 & 2.227770000 \\
\hline 1 & 5.613361000 & -1.515385000 & 2.105309000 \\
\hline 1 & 5.517193000 & 0.259184000 & 2.242956000 \\
\hline 1 & 4.448078000 & -0.744125000 & 3.212146000 \\
\hline 6 & -2.937216000 & -1.115124000 & 1.349059000 \\
\hline 0 & -2.110111000 & -1.859712000 & 0.488711000 \\
\hline 6 & -2.740721000 & -2.838501000 & -0.492337000 \\
\hline 8 & -0.838133000 & -1.730221000 & 0.433487000 \\
\hline 19 & 0.565504000 & -0.815898000 & -1.695778000 \\
\hline 8 & 2.726071000 & -0.252505000 & -0.108420000 \\
\hline 16 & 4.225827000 & 0.070992000 & -0.315774000 \\
\hline 6 & 4.554226000 & 1.575603000 & 0.659709000 \\
\hline 6 & 5.143203000 & -1.117271000 & 0.718979000 \\
\hline 8 & 1.040207000 & -0.669028000 & 2.164571000 \\
\hline 1 & 0.278262000 & -1.016217000 & 1.636472000 \\
\hline 1 & 5.627183000 & 1.785680000 & 0.629925000 \\
\hline 1 & 4.003414000 & 2.392043000 & 0.187574000 \\
\hline 1 & 4.212606000 & 1.426791000 & 1.687540000 \\
\hline 1 & 6.206230000 & -0.861382000 & 0.688870000 \\
\hline 1 & 4.760910000 & -1.082234000 & 1.742737000 \\
\hline 1 & 4.987720000 & -2.107783000 & 0.285813000 \\
\hline 1 & 1.725 & -0.483191000 & 2000 \\
\hline 1 & -3.832080000 & -2.865351000 & -0.423550000 \\
\hline 1 & -2.460726000 & -2.581723000 & -1.522057000 \\
\hline 1 & -2.353386000 & -3.846859000 & -0.296434000 \\
\hline 1 & -3.957863000 & -1.456057000 & 1.495404000 \\
\hline 1 & -2.475 & -0.610658000 & 2.192743000 \\
\hline 6 & -3.410589000 & 0.830252000 & 0.328185000 \\
\hline 6 & -2.260741000 & 1.644926000 & 0.439815000 \\
\hline 6 & -1.219 & $1.863 ?$ & -0.499052000 \\
\hline 1 & -2.144994000 & 2.167441000 & 1.388225000 \\
\hline 6 & -0.150108000 & 2.888696000 & -0.120493000 \\
\hline 8 & -1.091649000 & 1.302297000 & -1.631908000 \\
\hline 1 & -0.311168000 & 3.343159000 & 0.862098000 \\
\hline 1 & 02000 & 2.409484000 & -0.127188000 \\
\hline 1 & -0.126218000 & 3.684481000 & -0.876161000 \\
\hline 6 & -3.900193000 & 0.343902000 & -1.019796000 \\
\hline 1 & -3.095560000 & -0.059899000 & -1.632595000 \\
\hline 1 & -4.691842000 & -0.403607000 & -0.906803000 \\
\hline 1 & -4.328430000 & 1.199330000 & -1.566331000 \\
\hline 6 & -4.537095000 & 1.167836000 & 1.294298000 \\
\hline 1 & -4.166446000 & 1.351688000 & 2.308123000 \\
\hline 1 & -5.030942000 & 2.089448000 & 0.950537000 \\
\hline 1 & -5.298798000 & 0.383464000 & 1.334732000 \\
\hline 6 & -2.610805000 & -1.486525000 & -0.877522000 \\
\hline 6 & -1.812987000 & -0.468136000 & -1.658702000 \\
\hline 6 & -2.588314000 & 0.580869000 & -2.414830000 \\
\hline 8 & -0.583670000 & -0.557639000 & -1.780465000 \\
\hline 19 & 0.679070000 & 1.692854000 & -0.584850000 \\
\hline 8 & 2.902373000 & 0.181699000 & -0.040598000 \\
\hline 16 & 4.297466000 & 0.497110000 & 0.554320000 \\
\hline 6 & 4.568192000 & -0.730731000 & 1.874035000 \\
\hline 6 & 5.510416000 & -0.136445000 & -0.649678000 \\
\hline 8 & 1.682776000 & -2.162923000 & -1.073870000 \\
\hline 1 & 0.818075000 & -1.751412000 & -1.281333000 \\
\hline 1 & 5.587049000 & -0.617124000 & 2.255425000 \\
\hline 1 & 3.848954000 & -0.514061000 & 2.666896000 \\
\hline 1 & 4.408912000 & -1.736356000 & 1.475838000 \\
\hline 1 & 6.513372000 & -0.021960000 & -0.229092000 \\
\hline & 5.295821000 & -1.185617000 & -0.869820000 \\
\hline
\end{tabular}

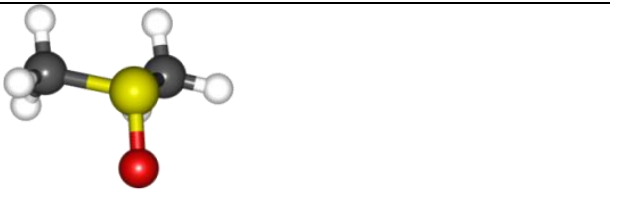

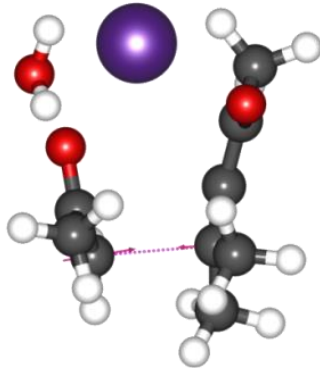

$\mathbf{T S}_{6 "} \rightarrow 7$

$\mathrm{E}(\mathrm{B} 2 \mathrm{PLYPD})=-1731.412187$ a.u., $i 334 \mathrm{~cm}^{-1}$

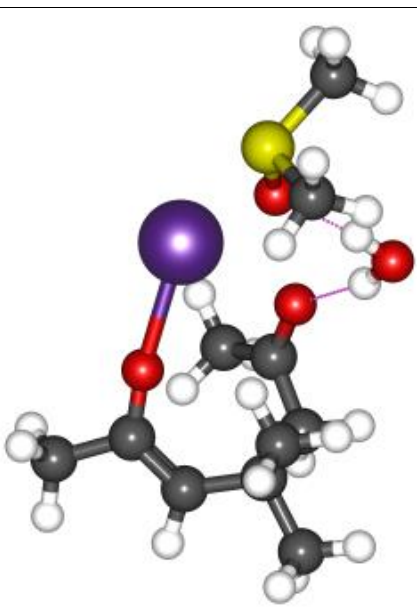

7 


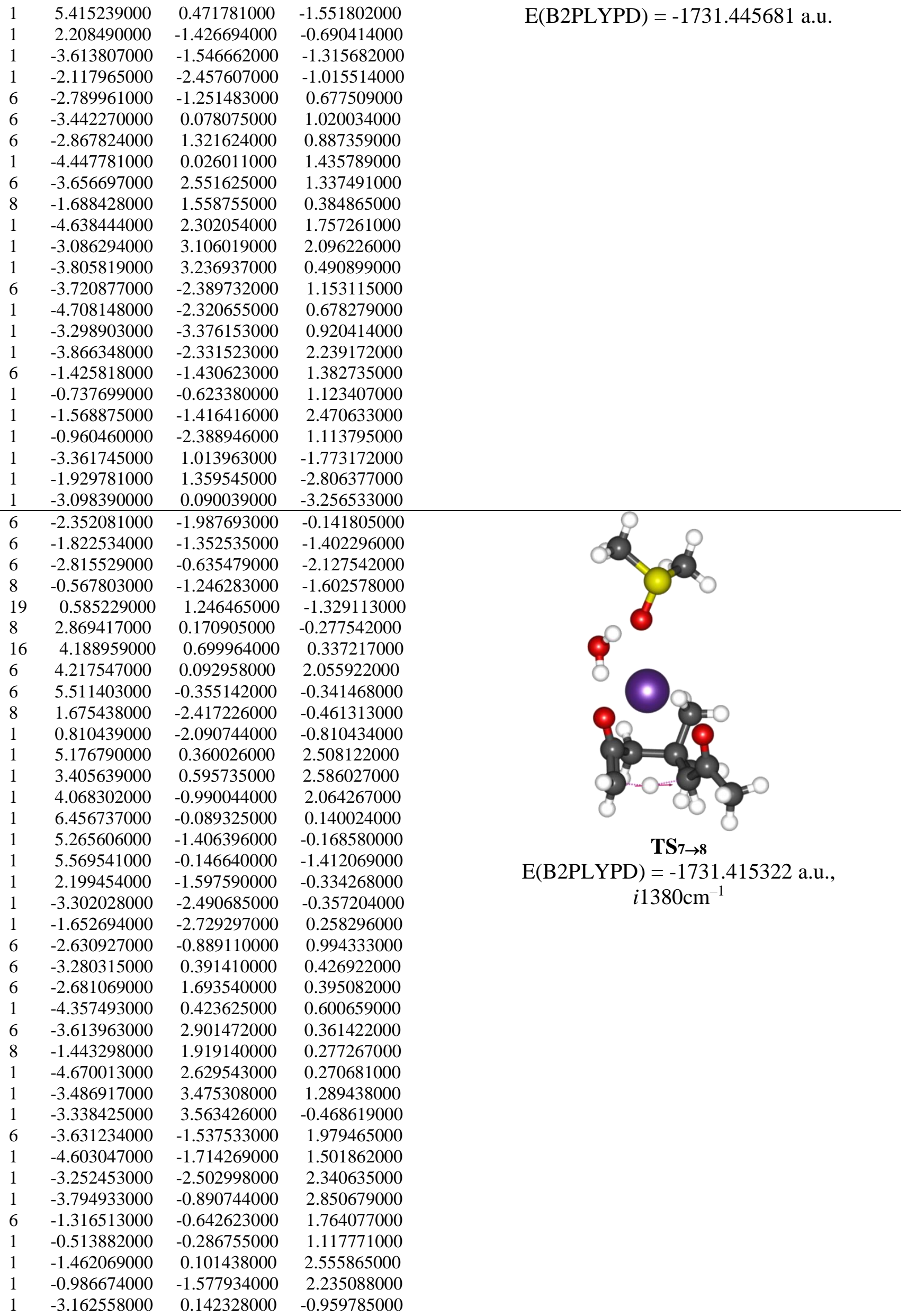




\begin{tabular}{|c|c|c|c|c|}
\hline 1 & -2.494381000 & -0.047877000 & -2.989340000 & \\
\hline 1 & -3.796393000 & -1.100263000 & -2.232799000 & \\
\hline 6 & -2.538934000 & -1.345101000 & -1.089783000 & \\
\hline 6 & -1.884994000 & -0.096677000 & -1.690224000 & \\
\hline 6 & -2.697362000 & 0.851153000 & -2.262031000 & \\
\hline 8 & -0.580824000 & -0.005370000 & -1.621288000 & \\
\hline 19 & 0.678511000 & 1.812705000 & -0.165869000 & \\
\hline 8 & 2.969770000 & 0.309049000 & -0.124874000 & \\
\hline 16 & 4.357333000 & 0.374047000 & 0.558256000 & \\
\hline 6 & 4.445403000 & -1.082100000 & 1.652473000 & \\
\hline 6 & 5.559386000 & -0.168648000 & -0.699813000 & \\
\hline 8 & 1.539756000 & -1.684195000 & -1.633397000 & \\
\hline 1 & 0.682026000 & -1.174501000 & -1.632915000 & \\
\hline 1 & 5.450053000 & -1.140759000 & 2.080752000 & \\
\hline 1 & 3.711886000 & -0.930966000 & 2.447573000 & 8 \\
\hline 1 & 4.210078000 & -1.983557000 & 1.080249000 & $\mathrm{E}(\mathrm{B} 2 \mathrm{PLYPD})=-1731.442512$ a.u. \\
\hline 1 & 6.548018000 & -0.232937000 & -0.236614000 & \\
\hline 1 & 5.253130000 & -1.136390000 & -1.106067000 & \\
\hline 1 & 5.567009000 & 0.590735000 & -1.484825000 & \\
\hline 1 & 2.147408000 & -1.111196000 & -1.121047000 & \\
\hline 1 & -3.549484000 & -1.448517000 & -1.506810000 & \\
\hline 1 & -1.967056000 & -2.225060000 & -1.418805000 & \\
\hline 6 & -2.668386000 & -1.429606000 & 0.463363000 & \\
\hline 6 & -3.445120000 & -0.208377000 & 1.028760000 & \\
\hline 6 & -2.839159000 & 1.188740000 & 1.019957000 & \\
\hline 1 & -3.657212000 & -0.400299000 & 2.094535000 & \\
\hline 6 & -3.826116000 & 2.332047000 & 0.894241000 & \\
\hline 8 & -1.649287000 & 1.409831000 & 1.236499000 & \\
\hline 1 & -4.375301000 & 2.259518000 & -0.049906000 & \\
\hline 1 & -4.564513000 & 2.267428000 & 1.705265000 & \\
\hline 1 & -3.309300000 & 3.293242000 & 0.951084000 & \\
\hline 6 & -3.532543000 & -2.673983000 & 0.778285000 & \\
\hline 1 & -4.542413000 & -2.581099000 & 0.358567000 & \\
\hline 1 & -3.074235000 & -3.576569000 & 0.354919000 & \\
\hline 1 & -3.628308000 & -2.823660000 & 1.861714000 & \\
\hline 6 & -1.302435000 & -1.628281000 & 1.148343000 & \\
\hline 1 & -0.604755000 & -0.828276000 & 0.906558000 & \\
\hline 1 & -1.419416000 & -1.670952000 & 2.239647000 & \\
\hline 1 & -0.859302000 & -2.577840000 & 0.821299000 & \\
\hline 1 & -4.426305000 & -0.155514000 & 0.541310000 & \\
\hline 1 & -2.278302000 & 1.762360000 & -2.687505000 & \\
\hline 1 & -3.776511000 & 0.729153000 & -2.296494000 & \\
\hline
\end{tabular}

Figure 3

\begin{tabular}{lccccc}
\hline 6 & -2.459878000 & 1.380018000 & 1.154621000 \\
6 & -1.882498000 & 0.025949000 & 1.536083000 \\
6 & -2.776231000 & -1.055943000 & 1.508040000 \\
8 & -0.617755000 & -0.107830000 & 1.662785000 \\
19 & 0.709986000 & -1.844270000 & 0.014147000 \\
8 & 2.955634000 & -0.271529000 & 0.017380000 \\
16 & 4.376996000 & -0.308677000 & -0.595507000 \\
6 & 4.597056000 & 1.286269000 & -1.450519000 \\
6 & 5.526995000 & -0.023769000 & 0.790440000 \\
8 & 1.554104000 & 1.599413000 & 1.666164000 \\
1 & 0.703965000 & 1.092567000 & 1.677546000 \\
1 & 5.626315000 & 1.348962000 & -1.814882000 \\
1 & 3.903602000 & 1.296876000 & -2.294212000 \\
1 & 4.375215000 & 2.105799000 & -0.761607000 \\
1 & 6.541056000 & 0.071149000 & 0.391700000 \\
1 & 5.231588000 & 0.880671000 & 1.329006000 \\
1 & 5.465320000 & -0.896548000 & 1.444123000
\end{tabular}




\subsection{0}

$-3.421714000$

$-1.786454000$

$-2.706201000$

$-3.457015000$

$-2.834208000$

$-3.587430000$

$-3.850645000$

$-1.660756000$

$-4.769089000$

$-4.121608000$

$-3.397407000$

$-3.603723000$

$-4.572973000$

$-3.122051000$

$-3.793760000$

$-1.377517000$

$-0.686554000$

$-1.560030000$

$-0.896698000$

$-4.466382000$

$-2.406975000$

$-3.831787000$

3.022529000

2.870055000

2.530328000

3.000657000

$-1.117070000$

$-3.294543000$

$-4.781023000$

$-5.736776000$

$-5.232177000$

$-1.169097000$

$-0.437603000$

$-6.802339000$

$-5.516141000$

$-5.433583000$

$-6.304224000$

$-4.972872000$

$-4.666477000$

$-1.981928000$

3.464311000

3.477128000

1.920031000

2.010366000

3.651039000

3.554686000

2.747863000

1.793856000

2.116732000

1.507887000

0.609955000

2.416363000

0.807351000

1.041512000

3.430901000

2.383682000

4.001806000

3.813697000

5.051624000

5.211318000

5.470717000
1.051926000

1.520666000

2.182233000

1.502500000

0.245820000

$-1.147554000$

0.339983000

$-2.293056000$

$-1.394318000$

$-2.101388000$

$-2.432213000$

$-3.224300000$

2.734436000

2.631040000

3.645700000

2.877537000

1.726326000

0.895189000

1.829897000

2.649526000

0.247716000

$-2.048581000$

$-0.876323000$

$-0.290367000$

1.207107000

1.706866000

1.971196000

$-1.479713000$

0.151633000

0.434968000

$-0.329043000$

$-0.727844000$

2.131042000

1.476598000

$-0.253338000$

0.235610000

$-1.373403000$

$-0.639137000$

$-1.746048000$

$-0.436073000$

1.586855000

$-0.417580000$

2.006604000

2.613362000

$-0.653415000$

$-0.493772000$

$-1.022944000$

$-0.606077000$

0.622273000

$-1.440520000$

1.192150000

0.260631000

1.541794000

2.033620000

0.417903000

$-2.541491000$

$-2.832622000$

$-2.858971000$

$-3.098850000$

$-0.682465000$

0.387352000

$-1.228008000$
1.106977000

1.664825000

1.478637000

$-0.386556000$

$-0.895718000$

$-0.666970000$

$-1.986549000$

$-0.747952000$

$-1.090207000$

$-0.185174000$

$-1.806301000$

$-0.394195000$

$-0.629449000$

$-0.125231000$

$-0.251404000$

$-1.701228000$

$-1.137230000$

$-0.991907000$

$-2.214959000$

$-0.788227000$

$-0.464685000$

1.759530000

1.689822000

$-1.302816000$

$-1.141486000$

0.245010000

$-2.097743000$

$-0.334211000$

$-0.305794000$

0.017973000

$-1.333220000$

1.347465000

$-0.194426000$

0.076787000

$-1.098377000$

$-2.241737000$

$-1.447642000$

1.544885000

1.044702000

2.235061000

$-0.222901000$

2.042368000

0.723925000

0.153819000

$-1.525190000$

$-2.178468000$

$-0.045867000$

1.230119000

1.079578000

1.560993000

2.489803000

0.442797000

3.000572000

2.417497000

3.113704000

$-0.278043000$

$-0.428272000$

$-1.161151000$

0.586863000

0.135215000

0.318633000

0.990098000
$\mathrm{E}(\mathrm{B} 2 \mathrm{PLYPD})=-1731.444054$ a.u., $i 261 \mathrm{~cm}^{-1}$ 


\begin{tabular}{|c|c|c|c|c|}
\hline 1 & 5.629368000 & -0.957919000 & -0.756962000 & \\
\hline 6 & -2.783607000 & 0.708259000 & -1.334842000 & \\
\hline 6 & -3.180934000 & -0.741781000 & -1.163561000 & \\
\hline 6 & -3.161732000 & -1.291778000 & 0.250236000 & \\
\hline 8 & -3.496081000 & -1.444743000 & -2.120200000 & \\
\hline 19 & 1.384968000 & -1.064202000 & -1.033019000 & \\
\hline 8 & 3.725004000 & 0.134158000 & -0.571305000 & \\
\hline 16 & 5.032496000 & 0.105211000 & 0.247585000 & \\
\hline 6 & 6.344876000 & 0.681754000 & -0.881056000 & \\
\hline 6 & 4.976889000 & 1.567472000 & 1.337193000 & \\
\hline 8 & 0.338144000 & -2.817423000 & 0.553558000 & \\
\hline 1 & -0.522853000 & -1.536341000 & 0.741775000 & \\
\hline 1 & 7.277295000 & 0.784192000 & -0.318537000 & \\
\hline 1 & 6.460903000 & -0.080228000 & -1.655058000 & \\
\hline 1 & 6.048449000 & 1.635949000 & -1.325731000 & (0) \\
\hline 1 & 5.931653000 & 1.655208000 & 1.863555000 & \\
\hline 1 & 4.776217000 & 2.459582000 & 0.737301000 & 4 a.u. \\
\hline 1 & 4.171339000 & 1.401914000 & 2.056079000 & \\
\hline 1 & 0.124875000 & -3.488079000 & 1.219330000 & \\
\hline 1 & -3.395040000 & 1.155492000 & 1.978237000 & \\
\hline 1 & -4.196048000 & -1.266911000 & 0.625442000 & \\
\hline 1 & -2.868767000 & -2.347196000 & 0.212558000 & \\
\hline 1 & -1.691401000 & 0.704551000 & -1.455531000 & \\
\hline 1 & -3.214370000 & 1.086674000 & -2.269213000 & \\
\hline 6 & -3.160054000 & 1.611739000 & -0.135515000 & \\
\hline 6 & -2.630933000 & 1.003716000 & 1.205556000 & \\
\hline 6 & -2.232018000 & -0.492196000 & 1.187076000 & \\
\hline 1 & -1.740970000 & 1.551016000 & 1.537391000 & \\
\hline 6 & -2.286384000 & -1.069275000 & 2.610888000 & \\
\hline 8 & -0.891946000 & -0.567877000 & 0.687515000 & \\
\hline 1 & -3.306725000 & -1.051633000 & 3.012514000 & \\
\hline 1 & -1.932778000 & -2.107184000 & 2.619743000 & \\
\hline 1 & -1.642542000 & -0.483337000 & 3.277882000 & \\
\hline 6 & -2.538598000 & 3.004674000 & -0.353937000 & \\
\hline 1 & -1.445962000 & 2.941634000 & -0.428850000 & \\
\hline 1 & -2.916911000 & 3.467828000 & -1.274445000 & \\
\hline 1 & -2.781127000 & 3.672971000 & 0.482218000 & \\
\hline 6 & -4.696970000 & 1.768049000 & -0.082711000 & \\
\hline 1 & -5.207079000 & 0.812934000 & 0.091345000 & \\
\hline 1 & -4.986572000 & 2.446964000 & 0.728826000 & \\
\hline 1 & -5.080088000 & 2.183769000 & -1.023351000 & \\
\hline 6 & -1.846176000 & 1.375664000 & -0.095766000 & \\
\hline 6 & -1.466060000 & 0.201207000 & -0.982371000 & \\
\hline 6 & -2.050673000 & -1.062113000 & -0.629018000 & \\
\hline 8 & -0.609112000 & 0.348322000 & -1.897337000 & \\
\hline 19 & 1.588752000 & -1.161396000 & -0.994727000 & \\
\hline 8 & 3.982624000 & -0.317884000 & -0.263916000 & \\
\hline 16 & 5.290100000 & -0.015925000 & 0.496489000 & \\
\hline 6 & 6.345204000 & 0.908776000 & -0.669499000 & \\
\hline 6 & 4.904138000 & 1.357515000 & 1.634845000 & \\
\hline 8 & -0.219002000 & -2.135951000 & 0.916864000 & \\
\hline 1 & -1.853777000 & -1.432146000 & 1.752626000 & \\
\hline 1 & 7.250445000 & 1.233869000 & -0.148921000 & \\
\hline 1 & 6.609007000 & 0.222154000 & -1.477138000 & \\
\hline 1 & 5.789527000 & 1.764948000 & -1.061999000 & \\
\hline 1 & 5.825816000 & 1.684965000 & 2.124583000 & \\
\hline 1 & 4.446431000 & 2.176292000 & 1.072549000 & \\
\hline 1 & 4.206257000 & 0.969980000 & 2.380524000 & \\
\hline 1 & -0.320155000 & -3.102921000 & 0.900534000 & \\
\hline 1 & -4.687341000 & 0.011989000 & -0.761213000 & TS10a $\rightarrow$ 11a \\
\hline 1 & -2.163944000 & -1.753379000 & -1.469940000 & $\mathrm{E}(\mathrm{B} 2 \mathrm{PLYPD})=-1731.437302$ a.u., \\
\hline 1 & -1.046066000 & -1.672395000 & 0.096265000 & $i 1277 \mathrm{~cm}^{-1}$ \\
\hline
\end{tabular}




\begin{tabular}{|c|c|c|c|}
\hline 1 & -1.424157000 & 1.183414000 & 0.899198000 \\
\hline 1 & -1.381765000 & 2.289231000 & -0.484113000 \\
\hline 6 & -3.385573000 & 1.567789000 & 0.050480000 \\
\hline ) & -4.119316000 & 0.190689000 & 0.160807000 \\
\hline 6 & -3.217250000 & -1.040509000 & 0.366365000 \\
\hline 1 & -4.855698000 & 0.227210000 & 0.975263000 \\
\hline 6 & -4.042393000 & -2.330295000 & 0.248108000 \\
\hline 8 & -2.711758000 & -0.952924000 & 1.736664000 \\
\hline 1 & -4.483434000 & -2.431104000 & -0.751304000 \\
\hline 1 & -3.406809000 & -3.206199000 & 0.429147000 \\
\hline 1 & -4.852433000 & -2.337015000 & 0.987361000 \\
\hline 6 & -3.654418000 & 2.423568000 & 1.303644000 \\
\hline 1 & -3.340526000 & 1.896225000 & 2.211593000 \\
\hline 1 & -3.108572000 & 3.375469000 & 1.254472000 \\
\hline 1 & -4.723623000 & 2.655503000 & 1.397381000 \\
\hline 6 & -3.915504000 & 2.322262000 & -1.187480000 \\
\hline 1 & -3.690202000 & 1.777980000 & -2.113748000 \\
\hline 1 & -5.004822000 & 2.446485000 & -1.131294000 \\
\hline 1 & -3.465965000 & 3.320935000 & -1.264736000 \\
\hline 6 & 2.859856000 & 1.338998000 & -0.694233000 \\
\hline 6 & 1.594428000 & 1.291017000 & 5000 \\
\hline 6 & 1.210084000 & 0.099182000 & 0.735821000 \\
\hline 8 & 0.952898000 & 2.419724000 & 0.288735000 \\
\hline 1 & 1.331451000 & -3.031785000 & -0.074905000 \\
\hline 1 & 3.842540000 & -1.936229000 & -0.142217000 \\
\hline 1 & 0.340215000 & 0.098276000 & 16000 \\
\hline 1 & 2.561487000 & 1.280279000 & -1.753618000 \\
\hline 1 & 3.317892000 & 2.328865000 & -0.562859000 \\
\hline 6 & 3.891009000 & 0.230382000 & -0.390738000 \\
\hline 6 & 3.138750000 & -1.120669000 & -0.364492000 \\
\hline 6 & 1.921057000 & -1.210530000 & 0.586919000 \\
\hline 1 & 2.761039000 & -1.300757000 & -1.379497000 \\
\hline 6 & 2.275339000 & & 1.946652000 \\
\hline 8 & 0.937870000 & -2.142159000 & 01000 \\
\hline 1 & 3.030111000 & -1.245613000 & 2.469748000 \\
\hline 1 & 1.382441000 & -1.894242000 & 2.580336000 \\
\hline 1 & 2.675060000 & -2.857617000 & 1.826876000 \\
\hline 6 & 4.947587000 & 0.181037000 & -1.511993000 \\
\hline 1 & 4.489012000 & -0.046950000 & -2.482874000 \\
\hline 1 & 5.465793000 & 1.144770000 & -1.603209000 \\
\hline 1 & 5.70 & 7464000 & 71000 \\
\hline 6 & 4.614539000 & 0.528973000 & 0.939088000 \\
\hline 1 & 3.910246000 & 0.691530000 & 1.760989000 \\
\hline 1 & 5.282771000 & -0.296012000 & 1.218854000 \\
\hline 1 & 5.225959000 & 1.435687000 & 0.842061000 \\
\hline 19 & -0.993025000 & -0.721985000 & -1.347446000 \\
\hline 8 & -3.076658000 & 0.421076000 & -0.058116000 \\
\hline 16 & -4.580415000 & 0.316644000 & 0.297456000 \\
\hline 6 & -5.442862000 & 0.011570000 & -1.278167000 \\
\hline 6 & -4.800472000 & -1.332330000 & 1.043278000 \\
\hline 8 & -1.603843000 & 2.610870000 & 1.133860000 \\
\hline 1 & -0.653162000 & 2.483099000 & 0.839827000 \\
\hline 1 & -6.501246000 & -0.168741000 & -1.070155000 \\
\hline 1 & -5.332789000 & 0.913567000 & -1.884092000 \\
\hline 1 & -4.991019000 & -0.847036000 & -1.782385000 \\
\hline 1 & -5.867032000 & -1.501810000 & 1.216726000 \\
\hline 1 & -4.386662000 & -2.092397000 & 0.374877000 \\
\hline 1 & -4.265911000 & -1.327619000 & 1.995779000 \\
\hline 1 & -2.107520000 & 1.870187000 & 0.737095000 \\
\hline
\end{tabular}




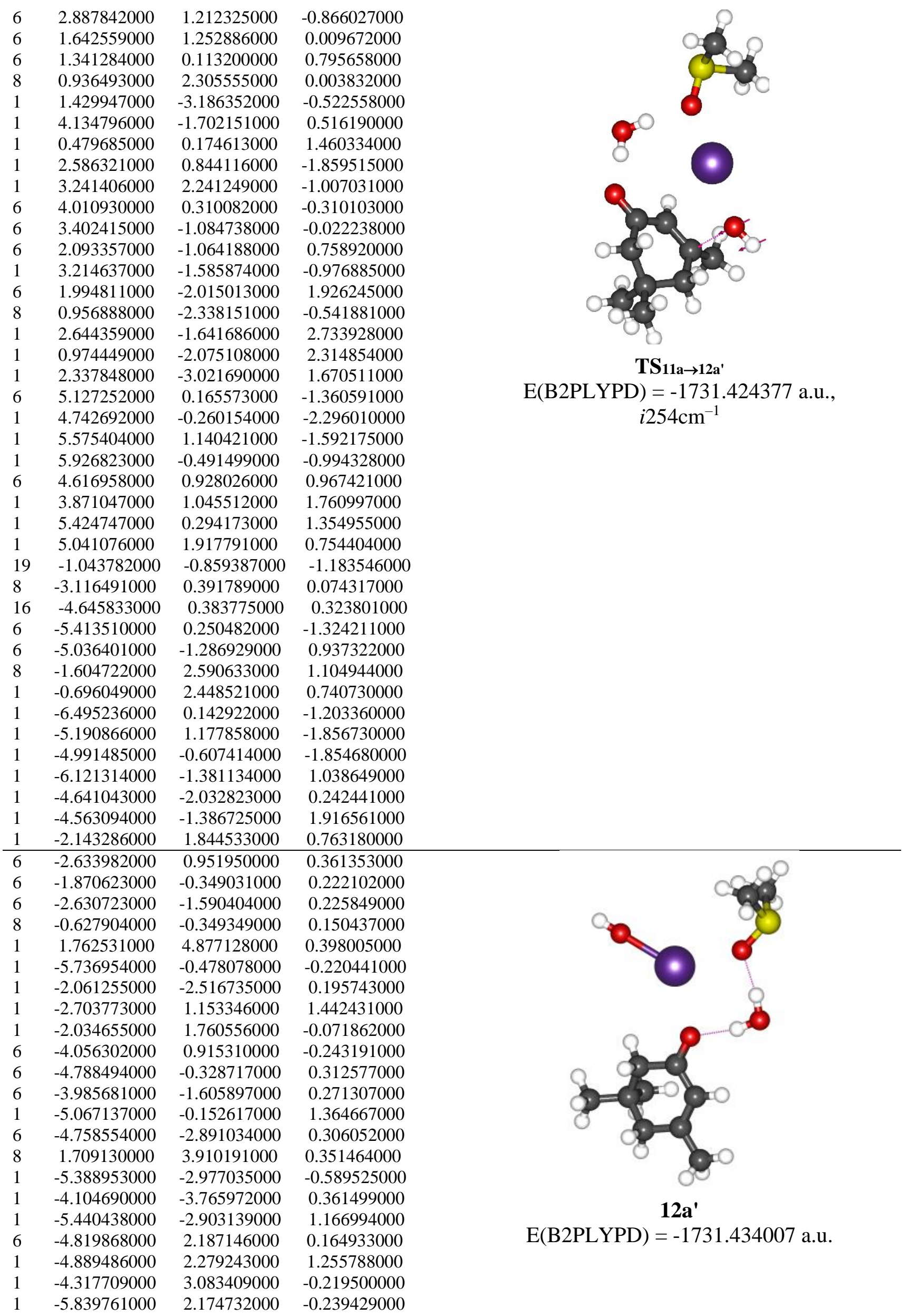




\begin{tabular}{|c|c|c|c|}
\hline 6 & -3.979086000 & 0.851398000 & -1.783569000 \\
\hline 1 & -3.455739000 & -0.044262000 & -2.137233000 \\
\hline 1 & -4.985876000 & 0.844011000 & -2.218614000 \\
\hline 1 & -3.448708000 & 1.726948000 & -2.177409000 \\
\hline 19 & 1.590165000 & 1.401407000 & 0.270930000 \\
\hline 8 & 3.383980000 & -0.669476000 & -0.040705000 \\
\hline 16 & 4.871472000 & -1.089232000 & -0.153350000 \\
\hline 6 & 5.553398000 & -0.119476000 & -1.537319000 \\
\hline 6 & 5.727871000 & -0.241615000 & 1.213607000 \\
\hline 8 & 1.250659000 & -2.516778000 & -0.088291000 \\
\hline 1 & 0.516761000 & -1.872256000 & -0.006385000 \\
\hline 1 & 6.632834000 & 08000 & -1.587664000 \\
\hline 1 & 5.077858000 & 27000 & -2.450235000 \\
\hline 1 & 5.328246000 & 0.939572000 & -1.384802000 \\
\hline 1 & 6.802890000 & -0.418976000 & 1.118627000 \\
\hline 1 & 5.502116000 & 46000 & 522000 \\
\hline 1 & 5.360685000 & 49000 & 2.143829000 \\
\hline 1 & 875000 & 6000 & -0.082518000 \\
\hline 6 & 2.219765000 & 67000 & 364000 \\
\hline 6 & 139000 & 20000 & -0.793903000 \\
\hline 6 & 1.758197000 & 0000 & -1.5 \\
\hline 8 & 0.690689000 & 26000 & 1960000 \\
\hline 19 & -1.554422000 & 58000 & 9885000 \\
\hline 8 & -3.094108000 & 44000 & -0.036507000 \\
\hline 16 & & & \\
\hline 6 & 649000 & -0.5 & 000 \\
\hline 6 & -5.556332000 & 76000 & -0.957232000 \\
\hline 8 & -0.649549000 & 53000 & 0.442714000 \\
\hline 1 & -0.055123000 & 84000 & -0.064662000 \\
\hline 1 & -6.240086000 & -0.804703000 & 1.596071000 \\
\hline 1 & 559000 & 3000 & 578000 \\
\hline 1 & -5.229081000 & 6000 & 0.57294 \\
\hline 1 & 4504000 & & \\
\hline 1 & -5.518985000 & 7000 & 0300000 \\
\hline 1 & -5.183787000 & 72000 & 3819000 \\
\hline 1 & 51000 & 2000 & 177000 \\
\hline 1 & 4.244849000 & -0.670753000 & -1.323948000 \\
\hline 1 & 2.435860000 & 1.630537000 & -1.277125000 \\
\hline 1 & 842000 & 1000 & 158000 \\
\hline 1 & 321000 & 32000 & 945000 \\
\hline 6 & & & \\
\hline 6 & 4.624584000 & -0.720584000 & -0.291907000 \\
\hline 6 & 5.271431000 & 0.630319000 & -0.036576000 \\
\hline 1 & 34000 & 7000 & 7446000 \\
\hline 6 & 6.519779000 & 0.914115000 & -0.853328000 \\
\hline 8 & & & \\
\hline 1 & 6.401658000 & 6000 & -1.892994000 \\
\hline 1 & 6.763371000 & 75000 & -0.819004000 \\
\hline 1 & 7.359775000 & 0.345217000 & -0.432218000 \\
\hline 6 & 3.991209000 & -1.276976000 & 2.103820000 \\
\hline 1 & 4.184652000 & 315000 & 2.490241000 \\
\hline 1 & 3.235942000 & -1.749631000 & 2.744928000 \\
\hline 1 & 4.916215000 & -1.862769000 & 2.193785000 \\
\hline 6 & & -2.690466000 & 0.20315 \\
\hline 1 & 2.880704000 & -2.737066000 & -0.844045000 \\
\hline 1 & 4.081426000 & -3.327705000 & 0.328449000 \\
\hline 1 & 2.386449000 & -3.112070000 & 0.810970000 \\
\hline 1 & 1.310751000 & 0.924160000 & -2.579771000 \\
\hline 6 & -2.142148000 & 3.736908000 & 0.372397000 \\
\hline 8 & -2.050517000 & 2.636243000 & -0.165218000 \\
\hline 6 & -1.924838000 & 3.907096000 & 1.857346000 \\
\hline 1 & -1.220102000 & 4.724098000 & 2.051941000 \\
\hline
\end{tabular}

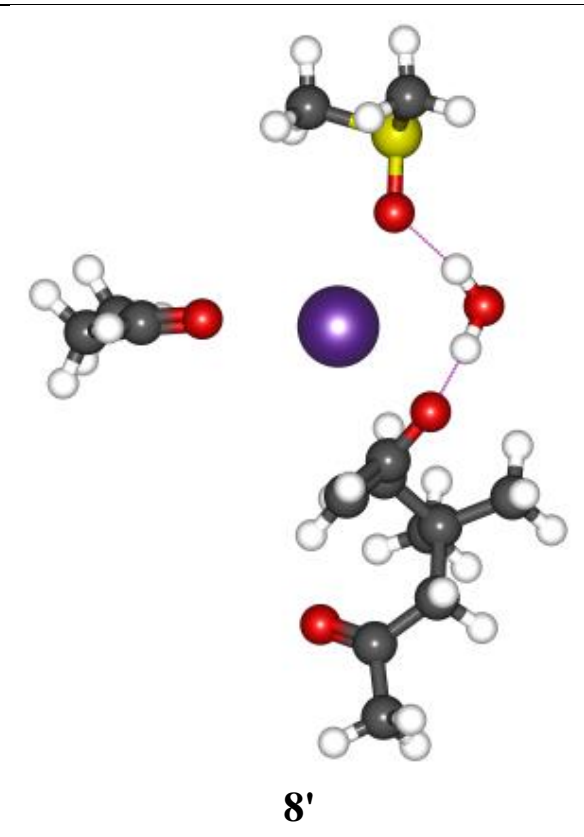

$\mathrm{E}(\mathrm{B} 2 \mathrm{PLYPD})=-1924.489388$ a.u. 


\begin{tabular}{|c|c|c|c|}
\hline 1 & -2.873937000 & 4.187644000 & 2.332734000 \\
\hline 1 & -1.556936000 & 2.980675000 & 2.303597000 \\
\hline 6 & -2.475291000 & 4.975810000 & -0.425014000 \\
\hline 1 & -3.290657000 & 5.533675000 & 0.050253000 \\
\hline 1 & -1.602384000 & 5.641660000 & -0.439488000 \\
\hline 1 & -2.747037000 & 4.714838000 & -1.450204000 \\
\hline 6 & 1.966380000 & -0.699421000 & 0.768632000 \\
\hline 6 & 1.168563000 & 0.078224000 & -0.276901000 \\
\hline 6 & 1.627760000 & 1.346064000 & -0.698798000 \\
\hline 8 & 0.059713000 & -0.411900000 & -0.677131000 \\
\hline 19 & -2.169841000 & 1.042134000 & -1.283604000 \\
\hline 8 & -3.872038000 & -0.755039000 & -0.107495000 \\
\hline 16 & -5.298342000 & -1.098453000 & 0000 \\
\hline 6 & -5.814147000 & 0.308355000 & 1.426540000 \\
\hline 6 & -6.398148000 & -0.795572000 & -1.032371000 \\
\hline 8 & -1.579719000 & -2.380039000 & 0.394831000 \\
\hline 1 & -0.896896000 & -1.745937000 & 0.062364000 \\
\hline 1 & -6.858382000 & 0.166408000 & 216000 \\
\hline 1 & -5.176488000 & 0.303913000 & 197000 \\
\hline 1 & -5.686965000 & 1.239171000 & 71000 \\
\hline 1 & -7.435725000 & -0.915186000 & 038000 \\
\hline 1 & -6.222034000 & 0.211769000 & -1.419440000 \\
\hline 1 & -6.157571000 & -1.544711000 & -1.789849000 \\
\hline 1 & -2.428683000 & -1.902469000 & 0.274892000 \\
\hline 1 & 3.549577000 & -0.727437000 & -1.568829000 \\
\hline 1 & 2.660610000 & 1.610547000 & -0.4 \\
\hline 1 & 2.564278000 & -0.007363000 & 1.366179000 \\
\hline 1 & 1.249290000 & -1.192193000 & 83000 \\
\hline 6 & 2.924473000 & -1.807964000 & 0.221839000 \\
\hline 6 & 4.019064000 & -1.240932000 & -0.715428000 \\
\hline 6 & 5.059922000 & -0.271484000 & -0.177245000 \\
\hline 1 & 4.572343000 & -2.077813000 & -1.167000000 \\
\hline 6 & 6.238269000 & -0.008183000 & \\
\hline 8 & 4.983761000 & 0.276723000 & 509000 \\
\hline 1 & 5.914591000 & 0.122790000 & -2.134860000 \\
\hline 1 & 6.792108000 & & -0.762016000 \\
\hline 1 & 6.908949000 & -0.877840000 & -1.076224000 \\
\hline 6 & 3.570367000 & -2.521370000 & 1.429078000 \\
\hline 1 & 4.107953000 & -1.819789000 & 2.073745000 \\
\hline 1 & 2.802560000 & -3.020761000 & 2.033142000 \\
\hline 1 & 4.279630000 & -3.289016000 & 1.091984000 \\
\hline 6 & 2.133768000 & -2.856339000 & -0.589704000 \\
\hline 1 & 1.696260000 & -2.425530000 & -1.496498000 \\
\hline 1 & 2.789555000 & -3.684395000 & -0.888742000 \\
\hline 1 & 1.315438000 & -3.272339000 & 0.009320000 \\
\hline 1 & 1.231997000 & 1.723437000 & -1.639062000 \\
\hline 6 & 0.543157000 & 2.794818000 & 0.454405000 \\
\hline 8 & -0.695512000 & 2.629790000 & 0.199067000 \\
\hline 6 & 1.018582000 & 2.479186000 & 1.873158000 \\
\hline 1 & 2.108890000 & 2.433306000 & 1.956892000 \\
\hline 1 & 0.660673000 & 3.281692000 & 2.537097000 \\
\hline 1 & 0.588414000 & 1.537136000 & 2.225235000 \\
\hline 6 & 1.232667000 & 4.011131000 & -0.172887000 \\
\hline 1 & 0.865276000 & 4.909719000 & 0.346533000 \\
\hline 1 & 2.322263000 & 3.985313000 & -0.072510000 \\
\hline 1 & 0.968855000 & 4.104337000 & -1.230847000 \\
\hline
\end{tabular}




$\begin{array}{lccc}6 & -2.688747000 & -0.810900000 & -1.008316000 \\ 6 & -1.539172000 & -0.495799000 & -0.058683000 \\ 6 & -1.424456000 & 0.883480000 & 0.525266000 \\ 8 & -0.700061000 & -1.366224000 & 0.198167000 \\ 19 & 2.015463000 & -0.880593000 & 0.561913000 \\ 8 & 4.545065000 & 0.094402000 & 0.075091000 \\ 16 & 6.055430000 & -0.177332000 & -0.122249000 \\ 6 & 6.695078000 & -0.647102000 & 1.519363000 \\ 6 & 6.165755000 & -1.811213000 & -0.924062000 \\ 8 & 3.174054000 & 2.533149000 & -0.712051000 \\ 1 & 2.243085000 & 2.165257000 & -0.554681000 \\ 1 & 7.740536000 & -0.951910000 & 1.417658000 \\ 1 & 6.627340000 & 0.238513000 & 2.154994000 \\ 1 & 6.088101000 & -1.459531000 & 1.928207000 \\ 1 & 7.218106000 & -2.100829000 & -0.995062000 \\ 1 & 5.596544000 & -2.541314000 & -0.342005000 \\ 1 & 5.742228000 & -1.705945000 & -1.925359000 \\ 1 & 3.759856000 & 1.783351000 & -0.481227000 \\ 1 & -3.799135000 & -0.692615000 & 1.616374000 \\ 1 & -2.406898000 & 1.366124000 & 0.552836000 \\ 1 & -3.068535000 & 0.114380000 & -1.446377000 \\ 1 & -2.283972000 & -1.437050000 & -1.813153000 \\ 6 & -3.894973000 & -1.588959000 & -0.380174000 \\ 6 & -4.540877000 & -0.845928000 & 0.816354000 \\ 6 & -5.208148000 & 0.505224000 & 0.600627000 \\ 1 & -5.296579000 & -1.502454000 & 1.271733000 \\ 6 & -6.085673000 & 0.987369000 & 1.738786000 \\ 8 & -5.063947000 & 1.178013000 & -0.413389000 \\ 1 & -5.624479000 & 0.788841000 & 2.712742000 \\ 1 & -6.295581000 & 2.054403000 & 1.632404000 \\ 1 & -7.034824000 & 0.434995000 & 1.714445000 \\ 6 & -4.939329000 & -1.813894000 & -1.494620000 \\ 1 & -5.281313000 & -0.868898000 & -1.926199000 \\ 1 & -4.513252000 & -2.423971000 & -2.300492000 \\ 1 & -5.813038000 & -2.347774000 & -1.099027000 \\ 6 & -3.434010000 & -2.969298000 & 0.134503000 \\ 1 & -2.718477000 & -2.884483000 & 0.958825000 \\ 1 & -4.295116000 & -3.548390000 & 0.490918000 \\ 1 & -2.953103000 & -3.540076000 & -0.668912000 \\ 1 & -1.032568000 & 0.800560000 & 1.544981000 \\ 6 & -0.399117000 & 1.837869000 & -0.260473000 \\ 8 & 0.868465000 & 1.315593000 & -0.224427000 \\ 6 & -0.889208000 & 2.040131000 & -1.718022000 \\ 1 & -1.921667000 & 2.411531000 & -1.775823000 \\ 1 & -0.237213000 & 2.769349000 & -2.215856000 \\ 1 & -0.826669000 & 1.101669000 & -2.281918000 \\ 6 & -0.474966000 & 3.205272000 & 0.470487000 \\ 1 & 0.210995000 & 3.911702000 & -0.014441000 \\ 1 & -1.483577000 & 3.641140000 & 0.453852000 \\ 1 & -0.163005000 & 3.095022000 & 1.516851000\end{array}$

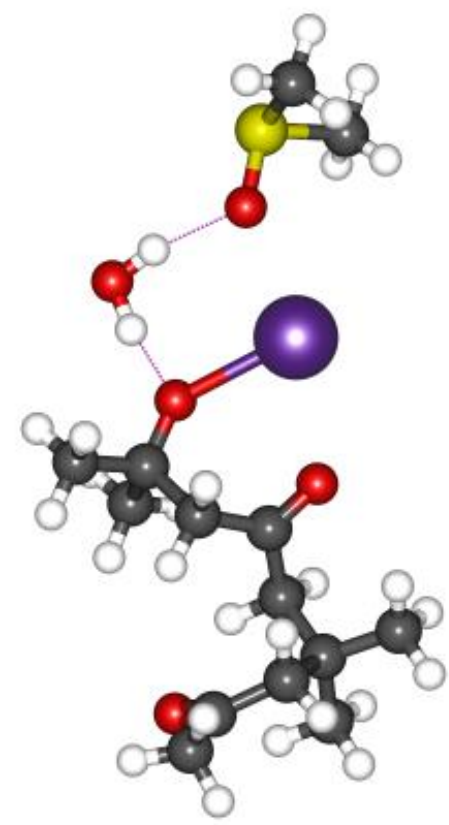

9b

$\mathrm{E}(\mathrm{B} 2 \mathrm{PLYPD})=-1924.502506$ a.u. 


$\begin{array}{lccc}6 & -3.023965000 & 0.117324000 & -0.552027000 \\ 6 & -1.780997000 & 0.687065000 & 0.108307000 \\ 8 & -0.776510000 & 0.014544000 & 0.336096000 \\ 6 & -1.855583000 & 2.144707000 & 0.529815000 \\ 6 & -1.033813000 & 3.149954000 & -0.355953000 \\ 6 & -1.407727000 & 3.028489000 & -1.839165000 \\ 6 & -1.346365000 & 4.573856000 & 0.145452000 \\ 8 & 0.358369000 & 2.891194000 & -0.260489000 \\ 19 & 1.817172000 & 0.659332000 & 1.043361000 \\ 8 & 3.831741000 & -0.877536000 & 0.169613000 \\ 16 & 5.335729000 & -1.211487000 & 0.093910000 \\ 6 & 5.932983000 & -0.501693000 & -1.477551000 \\ 6 & 5.437130000 & -2.972692000 & -0.371758000 \\ 1 & -2.482695000 & 3.174645000 & -1.996600000 \\ 1 & -1.126434000 & 2.047123000 & -2.237462000 \\ 1 & -0.870774000 & 3.789788000 & -2.416576000 \\ 1 & -2.902351000 & 2.468127000 & 0.543735000 \\ 1 & -1.462936000 & 2.217103000 & 1.550615000 \\ 1 & -3.817377000 & 0.189665000 & 0.204722000 \\ 1 & -3.322533000 & 0.824755000 & -1.339754000 \\ 1 & -0.758597000 & 5.301551000 & -0.426368000 \\ 1 & -2.408900000 & 4.818131000 & 0.025838000 \\ 1 & -1.086758000 & 4.680394000 & 1.205286000 \\ 1 & 6.972715000 & -0.804204000 & -1.631430000 \\ 1 & 5.298238000 & -0.848851000 & -2.297684000 \\ 1 & 5.873349000 & 0.584862000 & -1.382667000 \\ 1 & 6.484129000 & -3.233005000 & -0.551818000 \\ 1 & 5.051028000 & -3.551087000 & 0.470593000 \\ 1 & 4.830515000 & -3.147329000 & -1.264869000 \\ 1 & 1.447737000 & 3.903300000 & 2.462613000 \\ 8 & 1.450420000 & 3.025495000 & 2.052407000 \\ 1 & 0.715029000 & 3.063902000 & 0.702426000 \\ 6 & -3.002140000 & -1.315205000 & -1.139933000 \\ 6 & -1.970535000 & -1.402324000 & -2.286436000 \\ 1 & -2.186898000 & -0.651664000 & -3.057117000 \\ 1 & -0.950763000 & -1.237884000 & -1.925200000 \\ 1 & -2.007458000 & -2.389340000 & -2.764590000 \\ 6 & -4.402125000 & -1.604237000 & -1.725633000 \\ 1 & -4.436748000 & -2.612193000 & -2.158372000 \\ 1 & -5.182855000 & -1.533504000 & -0.961565000 \\ 1 & -4.642862000 & -0.890574000 & -2.523585000 \\ 6 & -2.637746000 & -2.399759000 & -0.095088000 \\ 1 & -1.620178000 & -2.221346000 & 0.279265000 \\ 1 & -2.590180000 & -3.376938000 & -0.597778000 \\ 6 & -3.506052000 & -2.575942000 & 1.138873000 \\ 8 & -4.341430000 & -1.756292000 & 1.506911000 \\ 6 & -3.273912000 & -3.852737000 & 1.923368000 \\ 1 & -3.717230000 & -3.774561000 & 2.919097000 \\ 1 & -3.745681000 & -4.689099000 & 1.389912000 \\ & -2.206100000 & -4.085354000 & 2.002990000\end{array}$

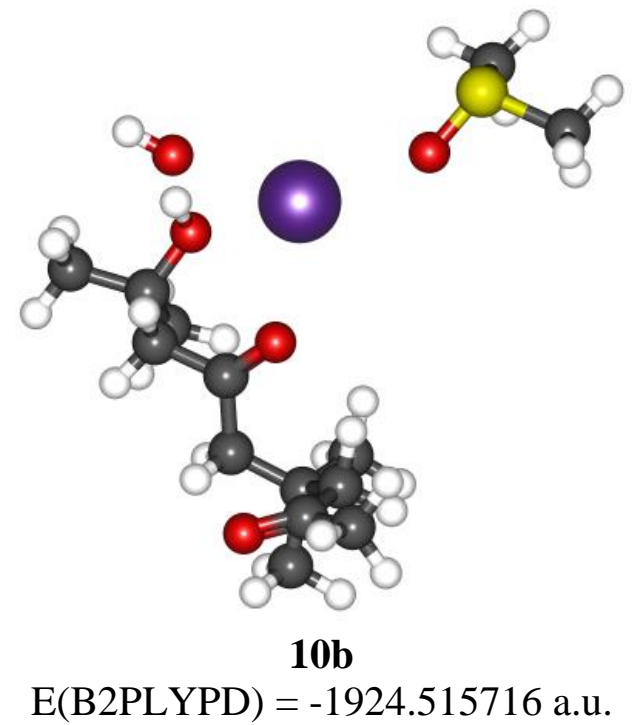

$\mathrm{E}(\mathrm{B} 2 \mathrm{PLYPD})=-1924.515716$ a.u. 


$\begin{array}{lccc}6 & 3.200320000 & -0.341893000 & 0.355764000 \\ 6 & 2.023021000 & 0.648708000 & 0.424238000 \\ 8 & 0.980852000 & 0.364823000 & 1.071769000 \\ 6 & 2.182358000 & 1.845395000 & -0.361777000 \\ 6 & 1.506210000 & 3.181080000 & 0.013240000 \\ 6 & 1.473530000 & 3.467672000 & 1.519354000 \\ 6 & 2.237982000 & 4.323863000 & -0.714350000 \\ 8 & 0.107109000 & 3.208125000 & -0.414987000 \\ 19 & -1.300044000 & 0.630242000 & -0.397778000 \\ 8 & -3.806764000 & -0.170345000 & -0.064080000 \\ 16 & -5.341959000 & -0.291841000 & -0.148567000 \\ 6 & -5.990229000 & 0.443130000 & 1.390756000 \\ 6 & -5.728325000 & -2.036242000 & 0.221369000 \\ 1 & 2.494490000 & 3.544471000 & 1.911563000 \\ 1 & 0.955246000 & 2.669432000 & 2.056723000 \\ 1 & 0.958949000 & 4.417321000 & 1.715567000 \\ 1 & 3.211991000 & 1.988388000 & -0.702685000 \\ 1 & 1.443175000 & 1.487738000 & -1.524306000 \\ 1 & 3.494877000 & -0.427169000 & -0.695998000 \\ 1 & 4.048136000 & 0.149799000 & 0.857781000 \\ 1 & 1.731865000 & 5.277806000 & -0.524506000 \\ 1 & 3.277279000 & 4.409002000 & -0.373706000 \\ 1 & 2.249898000 & 4.149113000 & -1.797665000 \\ 1 & -7.072504000 & 0.289553000 & 1.430866000 \\ 1 & -5.496130000 & -0.019391000 & 2.249888000 \\ 1 & -5.769171000 & 1.512241000 & 1.353516000 \\ 1 & -6.814128000 & -2.156967000 & 0.273762000 \\ 1 & -5.328477000 & -2.634619000 & -0.600373000 \\ 1 & -5.256403000 & -2.320285000 & 1.166125000 \\ 1 & 0.944127000 & 1.493667000 & -3.235822000 \\ 8 & 0.543199000 & 1.367281000 & -2.360610000 \\ 1 & 0.105083000 & 2.839598000 & -1.328713000 \\ 6 & 3.057550000 & -1.760761000 & 0.962729000 \\ 6 & 2.921267000 & -1.668058000 & 2.499384000 \\ 1 & 3.778864000 & -1.131561000 & 2.926449000 \\ 1 & 2.009136000 & -1.136720000 & 2.784565000 \\ 1 & 2.897424000 & -2.669042000 & 2.950495000 \\ 6 & 4.347890000 & -2.549159000 & 0.645414000 \\ 1 & 4.298884000 & -3.560388000 & 1.070847000 \\ 1 & 4.511616000 & -2.639575000 & -0.433241000 \\ 1 & 5.222567000 & -2.048358000 & 1.080076000 \\ 6 & 1.825127000 & -2.534649000 & 0.433111000 \\ 1 & 0.915632000 & -1.972983000 & 0.690036000 \\ 1 & 1.736855000 & -3.488741000 & 0.974198000 \\ 6 & 1.712504000 & -2.855306000 & -1.045899000 \\ 8 & 2.402309000 & -2.332663000 & -1.915360000 \\ 6 & 0.661110000 & -3.887719000 & -1.410001000 \\ 1 & 0.445293000 & -3.851952000 & -2.480738000 \\ 1 & 1.042129000 & -4.887007000 & -1.159045000 \\ 1 & -0.260051000 & -3.742653000 & -0.834632000\end{array}$

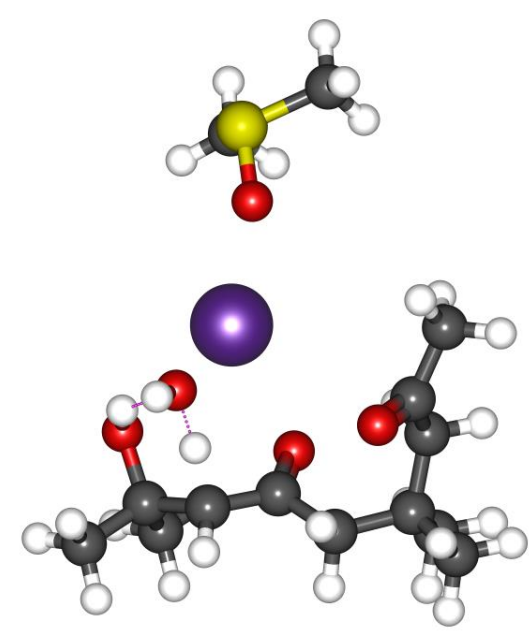

TS $10 \mathrm{~b} \rightarrow 11 \mathrm{~b}$

$\mathrm{E}(\mathrm{B} 2 \mathrm{PLYPD})=-1924.499536$ a.u., $i 1304 \mathrm{~cm}^{-1}$ 


$\begin{array}{lccc}6 & -2.112306000 & -0.974165000 & -1.271103000 \\ 6 & -1.252958000 & 0.235465000 & -0.875817000 \\ 8 & 0.030692000 & 0.030756000 & -0.749280000 \\ 6 & -1.884357000 & 1.447540000 & -0.681843000 \\ 6 & -1.222321000 & 2.748921000 & -0.301284000 \\ 6 & -0.135299000 & 3.208417000 & -1.286007000 \\ 6 & -2.275728000 & 3.857120000 & -0.141681000 \\ 8 & -0.514262000 & 2.628662000 & 0.998187000 \\ 19 & 1.810863000 & 1.055891000 & 0.910015000 \\ 8 & 3.816338000 & -0.532090000 & -0.101398000 \\ 16 & 5.332085000 & -0.832524000 & -0.199551000 \\ 6 & 5.754851000 & -1.753558000 & 1.315777000 \\ 6 & 6.173945000 & 0.732847000 & 0.202582000 \\ 1 & -0.595704000 & 3.498975000 & -2.236988000 \\ 1 & 0.573456000 & 2.400582000 & -1.485833000 \\ 1 & 0.407524000 & 4.077084000 & -0.890001000 \\ 1 & -2.963289000 & 1.482108000 & -0.819777000 \\ 1 & 1.079723000 & -1.086619000 & -1.536305000 \\ 1 & -3.029853000 & -0.632966000 & -1.759935000 \\ 1 & -1.540943000 & -1.551408000 & -2.012372000 \\ 1 & -1.801842000 & 4.797100000 & 0.164559000 \\ 1 & -2.807646000 & 4.028347000 & -1.085595000 \\ 1 & -3.017047000 & 3.579639000 & 0.618363000 \\ 1 & 6.838538000 & -1.897724000 & 1.350031000 \\ 1 & 5.405619000 & -1.196312000 & 2.189333000 \\ 1 & 5.253577000 & -2.721967000 & 1.253596000 \\ 1 & 7.250479000 & 0.549061000 & 0.259898000 \\ 1 & 5.959472000 & 1.431445000 & -0.609088000 \\ 1 & 5.791287000 & 1.118102000 & 1.151749000 \\ 1 & 2.606217000 & -1.314972000 & -1.356214000 \\ 8 & 1.846723000 & -1.610584000 & -1.901068000 \\ 1 & -1.104241000 & 2.107224000 & 1.568700000 \\ 6 & -2.525270000 & -1.968558000 & -0.138405000 \\ 6 & -1.280829000 & -2.554854000 & 0.560974000 \\ 1 & -0.625912000 & -3.047980000 & -0.167063000 \\ 1 & -0.694324000 & -1.776879000 & 1.059457000 \\ 1 & -1.576573000 & -3.301904000 & 1.310035000 \\ 6 & -3.306991000 & -3.137183000 & -0.777302000 \\ 1 & -3.641576000 & -3.849708000 & -0.010889000 \\ 1 & -4.186870000 & -2.784207000 & -1.323290000 \\ 1 & -2.665423000 & -3.683941000 & -1.480277000 \\ 6 & -3.377793000 & -1.286547000 & 0.958724000 \\ 1 & -2.816489000 & -0.439067000 & 1.381244000 \\ 1 & -3.512397000 & -1.982902000 & 1.800671000 \\ 6 & -4.755465000 & -0.733904000 & 0.633044000 \\ 8 & -5.198744000 & -0.609667000 & -0.503170000 \\ 6 & -5.590672000 & -0.330357000 & 1.835402000 \\ 1 & -6.424952000 & 0.303238000 & 1.524121000 \\ 1 & -5.992756000 & -1.235681000 & 2.310165000 \\ 1 & -4.986610000 & 0.186065000 & 2.589704000\end{array}$

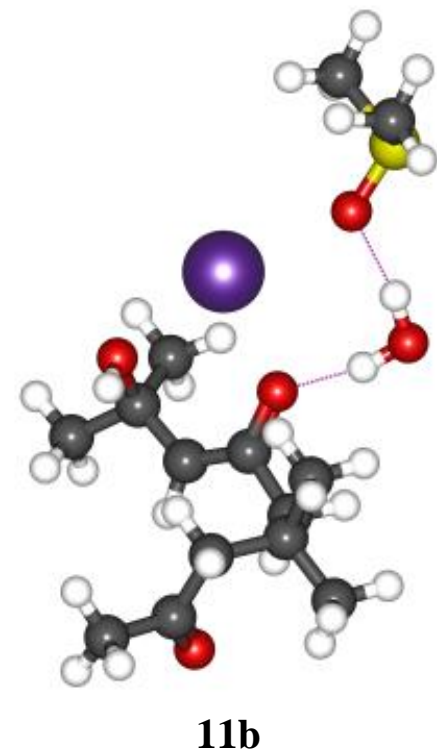

$\mathrm{E}(\mathrm{B} 2 \mathrm{PLYPD})=-1924.500987$ a.u. 


$\begin{array}{lccc}6 & -2.249380000 & -1.001520000 & -1.397047000 \\ 6 & -1.277338000 & 0.149347000 & -1.133104000 \\ 8 & -0.035924000 & -0.087081000 & -1.171345000 \\ 6 & -1.843150000 & 1.431656000 & -0.844796000 \\ 6 & -1.191004000 & 2.655505000 & -0.740190000 \\ 6 & 0.135555000 & 2.951536000 & -1.393136000 \\ 6 & -2.045783000 & 3.878883000 & -0.505139000 \\ 8 & -0.246800000 & 2.680827000 & 1.363362000 \\ 19 & 1.555417000 & 0.835659000 & 0.952546000 \\ 8 & 3.675098000 & -0.595357000 & -0.107986000 \\ 16 & 5.216999000 & -0.746857000 & -0.105324000 \\ 6 & 5.624026000 & -1.610961000 & 1.447360000 \\ 6 & 5.871382000 & 0.897869000 & 0.328434000 \\ 1 & -0.064763000 & 3.423146000 & -2.368118000 \\ 1 & 0.723506000 & 2.052930000 & -1.575009000 \\ 1 & 0.710522000 & 3.664076000 & -0.796660000 \\ 1 & -2.913092000 & 1.430923000 & -0.646109000 \\ 1 & 1.142343000 & -1.375157000 & -1.755633000 \\ 1 & -3.193563000 & -0.602124000 & -1.776472000 \\ 1 & -1.801916000 & -1.621142000 & -2.184729000 \\ 1 & -1.464113000 & 4.688837000 & -0.058604000 \\ 1 & -2.434423000 & 4.239534000 & -1.470164000 \\ 1 & -2.904541000 & 3.661121000 & 0.137861000 \\ 1 & 6.711706000 & -1.656325000 & 1.552258000 \\ 1 & 5.170694000 & -1.079508000 & 2.288543000 \\ 1 & 5.216759000 & -2.621366000 & 1.368152000 \\ 1 & 6.955296000 & 0.824932000 & 0.455578000 \\ 1 & 5.639843000 & 1.565765000 & -0.504124000 \\ 1 & 5.391990000 & 1.248446000 & 1.246535000 \\ 1 & 2.634524000 & -1.501981000 & -1.374911000 \\ 8 & 1.953117000 & -1.890514000 & -1.966382000 \\ 1 & -1.090213000 & 2.719136000 & 1.842195000 \\ 6 & -2.571572000 & -1.948355000 & -0.193959000 \\ 6 & -1.289372000 & -2.652951000 & 0.297005000 \\ 1 & -0.798764000 & -3.182637000 & -0.527860000 \\ 1 & -0.566985000 & -1.944002000 & 0.713760000 \\ 1 & -1.531604000 & -3.388291000 & 1.074934000 \\ 6 & -3.553944000 & -3.030083000 & -0.692698000 \\ 1 & -3.826261000 & -3.710815000 & 0.124555000 \\ 1 & -4.472621000 & -2.590259000 & -1.092245000 \\ 1 & -3.089893000 & -3.630288000 & -1.485167000 \\ 6 & -3.172060000 & -1.199164000 & 1.021812000 \\ 1 & -2.474534000 & -0.419308000 & 1.365881000 \\ 1 & -3.243085000 & -1.896821000 & 1.869083000 \\ 6 & -4.525510000 & -0.514887000 & 0.903214000 \\ 8 & -5.075635000 & -0.274255000 & -0.165360000 \\ 6 & -5.176535000 & -0.129709000 & 2.217477000 \\ 1 & -5.990556000 & 0.578969000 & 2.046725000 \\ 1 & -5.585222000 & -1.032698000 & 2.691009000 \\ 1 & -4.446683000 & 0.295141000 & 2.915848000\end{array}$

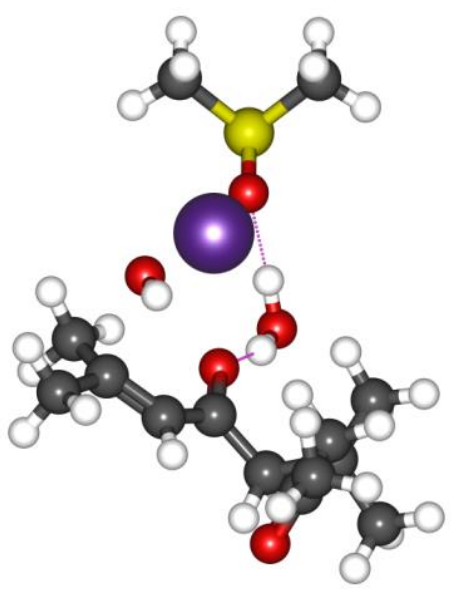

$\mathbf{T S}_{11 \mathrm{~b} \rightarrow \mathbf{1 2}^{\prime}}$

$\mathrm{E}(\mathrm{B} 2 \mathrm{PLYPD})=-1924.478299$ a.u., $i 212 \mathrm{~cm}^{-1}$ 


$\begin{array}{lccc}6 & -2.203912000 & -1.046788000 & -1.213967000 \\ 6 & -1.463993000 & 0.255907000 & -0.945576000 \\ 8 & -0.228709000 & 0.246744000 & -0.786728000 \\ 6 & -2.274104000 & 1.477763000 & -0.899898000 \\ 6 & -1.843471000 & 2.765529000 & -0.973071000 \\ 6 & -0.411416000 & 3.201302000 & -1.112928000 \\ 6 & -2.846445000 & 3.887462000 & -0.936031000 \\ 8 & 1.918276000 & 2.281370000 & 3.084296000 \\ 19 & 1.893090000 & 0.847373000 & 1.029312000 \\ 8 & 3.777236000 & -0.557294000 & -0.384073000 \\ 16 & 5.286889000 & -0.853278000 & -0.572698000 \\ 6 & 5.794144000 & -1.781423000 & 0.911194000 \\ 6 & 6.141429000 & 0.714417000 & -0.210586000 \\ 1 & -0.348318000 & 4.090777000 & -1.750628000 \\ 1 & 0.231239000 & 2.415051000 & -1.509828000 \\ 1 & -0.017313000 & 3.493405000 & -0.128484000 \\ 1 & -3.347734000 & 1.310640000 & -0.863979000 \\ 1 & 0.986573000 & -0.972389000 & -1.677226000 \\ 1 & -3.116660000 & -0.838366000 & -1.777315000 \\ 1 & -1.548326000 & -1.665940000 & -1.837313000 \\ 1 & -2.583472000 & 4.602052000 & -0.144277000 \\ 1 & -2.822182000 & 4.449442000 & -1.879689000 \\ 1 & -3.866682000 & 3.532699000 & -0.765715000 \\ 1 & 6.878015000 & -1.924606000 & 0.883083000 \\ 1 & 5.494125000 & -1.228738000 & 1.805570000 \\ 1 & 5.290597000 & -2.749880000 & 0.871842000 \\ 1 & 7.220461000 & 0.536455000 & -0.214647000 \\ 1 & 5.877960000 & 1.415446000 & -1.005534000 \\ 1 & 5.810611000 & 1.093163000 & 0.760282000 \\ 1 & 2.514616000 & -1.199127000 & -1.560532000 \\ 8 & 1.727836000 & -1.459959000 & -2.090710000 \\ 1 & 1.921016000 & 2.843483000 & 3.874324000 \\ 6 & -2.585063000 & -1.899518000 & 0.045231000 \\ 6 & -1.311365000 & -2.341846000 & 0.796046000 \\ 1 & -0.622273000 & -2.862387000 & 0.120950000 \\ 1 & -0.776697000 & -1.492927000 & 1.234941000 \\ 1 & -1.570889000 & -3.030124000 & 1.609781000 \\ 6 & -3.319055000 & -3.160991000 & -0.456186000 \\ 1 & -3.615897000 & -3.793439000 & 0.390161000 \\ 1 & -4.217979000 & -2.906617000 & -1.025515000 \\ 1 & -2.662065000 & -3.755564000 & -1.102562000 \\ 6 & -3.471051000 & -1.134248000 & 1.061389000 \\ 1 & -2.951707000 & -0.228830000 & 1.414222000 \\ 1 & -3.582141000 & -1.752606000 & 1.962976000 \\ 6 & -4.866492000 & -0.679694000 & 0.656630000 \\ 8 & -5.230481000 & -0.578255000 & -0.509503000 \\ 6 & -5.798928000 & -0.337745000 & 1.800154000 \\ 1 & -6.674862000 & 0.200913000 & 1.431032000 \\ 1 & -6.127760000 & -1.267309000 & 2.284053000 \\ & -5.286125000 & 0.256073000 & 2.565610000\end{array}$

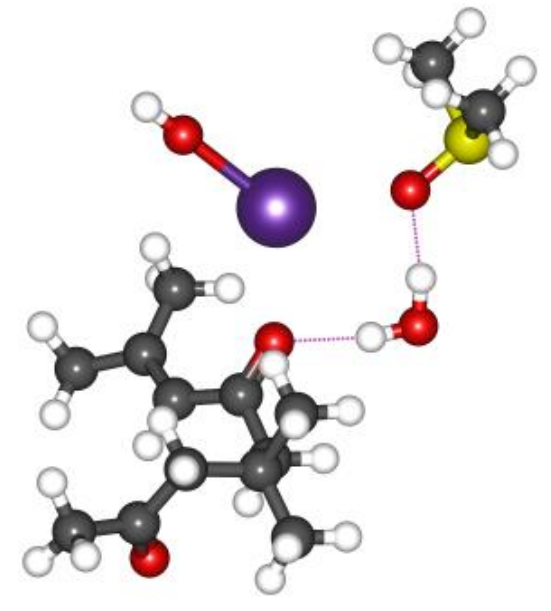

12b'

$\mathrm{E}(\mathrm{B} 2 \mathrm{PLYPD})=-1924.478408$ a.u. 


$\begin{array}{lrrr}6 & -2.236925000 & -1.075217000 & -1.256241000 \\ 6 & -1.489154000 & 0.236655000 & -1.034012000 \\ 8 & -0.258261000 & 0.230748000 & -0.899610000 \\ 6 & -2.305142000 & 1.463878000 & -1.000867000 \\ 6 & -1.864412000 & 2.748570000 & -0.983813000 \\ 6 & -0.419182000 & 3.170616000 & -0.988751000 \\ 6 & -2.858740000 & 3.879557000 & -0.956799000 \\ 1 & -0.308907000 & 4.151658000 & -1.464478000 \\ 1 & 0.225424000 & 2.441160000 & -1.480532000 \\ 1 & -0.058878000 & 3.272441000 & 0.045476000 \\ 1 & -3.380341000 & 1.301844000 & -1.025970000 \\ 1 & -3.163954000 & -0.885043000 & -1.803270000 \\ 1 & -1.591406000 & -1.706354000 & -1.878520000 \\ 1 & -2.653143000 & 4.544810000 & -0.106746000 \\ 1 & -2.758447000 & 4.495414000 & -1.861195000 \\ 1 & -3.892132000 & 3.528092000 & -0.885892000 \\ 6 & -2.582613000 & -1.894126000 & 0.032510000 \\ 6 & -1.287787000 & -2.298444000 & 0.769631000 \\ 1 & -0.614302000 & -2.843575000 & 0.097559000 \\ 1 & -0.744307000 & -1.429057000 & 1.154385000 \\ 1 & -1.521602000 & -2.954189000 & 1.617746000 \\ 6 & -3.310433000 & -3.181168000 & -0.410138000 \\ 1 & -3.586912000 & -3.786315000 & 0.462905000 \\ 1 & -4.220824000 & -2.958212000 & -0.974511000 \\ 1 & -2.656049000 & -3.791266000 & -1.044709000 \\ 6 & -3.456449000 & -1.106894000 & 1.042078000 \\ 1 & -2.941128000 & -0.184722000 & 1.355231000 \\ 1 & -3.543491000 & -1.696408000 & 1.965727000 \\ 6 & -4.864903000 & -0.679716000 & 0.654410000 \\ 8 & -5.263869000 & -0.633645000 & -0.503721000 \\ 6 & -5.769805000 & -0.295048000 & 1.807304000 \\ 1 & -6.647282000 & 0.243161000 & 1.440969000 \\ 1 & -6.100659000 & -1.208431000 & 2.320152000 \\ 1 & -5.236316000 & 0.311793000 & 2.547674000\end{array}$

Figure 4

\begin{tabular}{lccc}
\hline 6 & 2.345926000 & 2.529106000 & 0.659968000 \\
6 & 2.442228000 & 1.244986000 & 0.166455000 \\
8 & 1.453577000 & 0.604158000 & -0.414819000 \\
19 & -0.246567000 & -0.999975000 & 0.828926000 \\
8 & -2.491757000 & 0.076463000 & -0.240646000 \\
16 & -4.033688000 & 0.202053000 & -0.308249000 \\
6 & -4.649529000 & -1.470224000 & -0.689179000 \\
6 & -4.599441000 & 0.305953000 & 1.421451000 \\
8 & -0.659431000 & 1.822948000 & -1.604720000 \\
1 & 0.160673000 & 1.456133000 & -1.163807000 \\
1 & -5.742426000 & -1.461600000 & -0.648675000 \\
1 & -4.318186000 & -1.710692000 & -1.701870000 \\
1 & -4.236263000 & -2.183144000 & 0.029432000 \\
1 & -5.692991000 & 0.290613000 & 1.436114000 \\
1 & -4.187746000 & -0.531993000 & 1.990805000 \\
1 & -4.236743000 & 1.255569000 & 1.820950000 \\
1 & 3.191726000 & 3.020484000 & 1.133305000 \\
1 & 1.416990000 & 3.089389000 & 0.579040000 \\
1 & -1.390264000 & 1.301748000 & -1.211458000 \\
6 & 3.769305000 & 0.578208000 & 0.331951000 \\
1 & 4.484402000 & 1.183224000 & 0.892037000 \\
6 & 4.219143000 & -0.622917000 & -0.098693000 \\
6 & 3.434375000 & -1.627780000 & -0.908131000 \\
1 & 3.363747000 & -2.584972000 & -0.368795000 \\
1 & 2.431091000 & -1.266447000 & -1.132146000
\end{tabular}

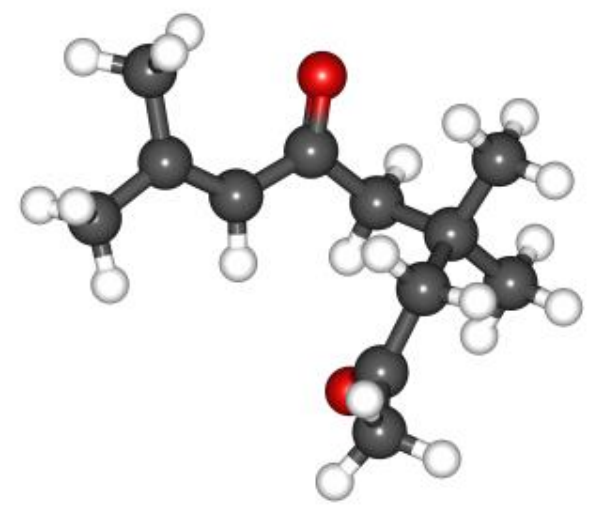

$12 b$

$\mathrm{E}(\mathrm{B} 2 \mathrm{PLYPD})=-619.3956344$ a.u. 


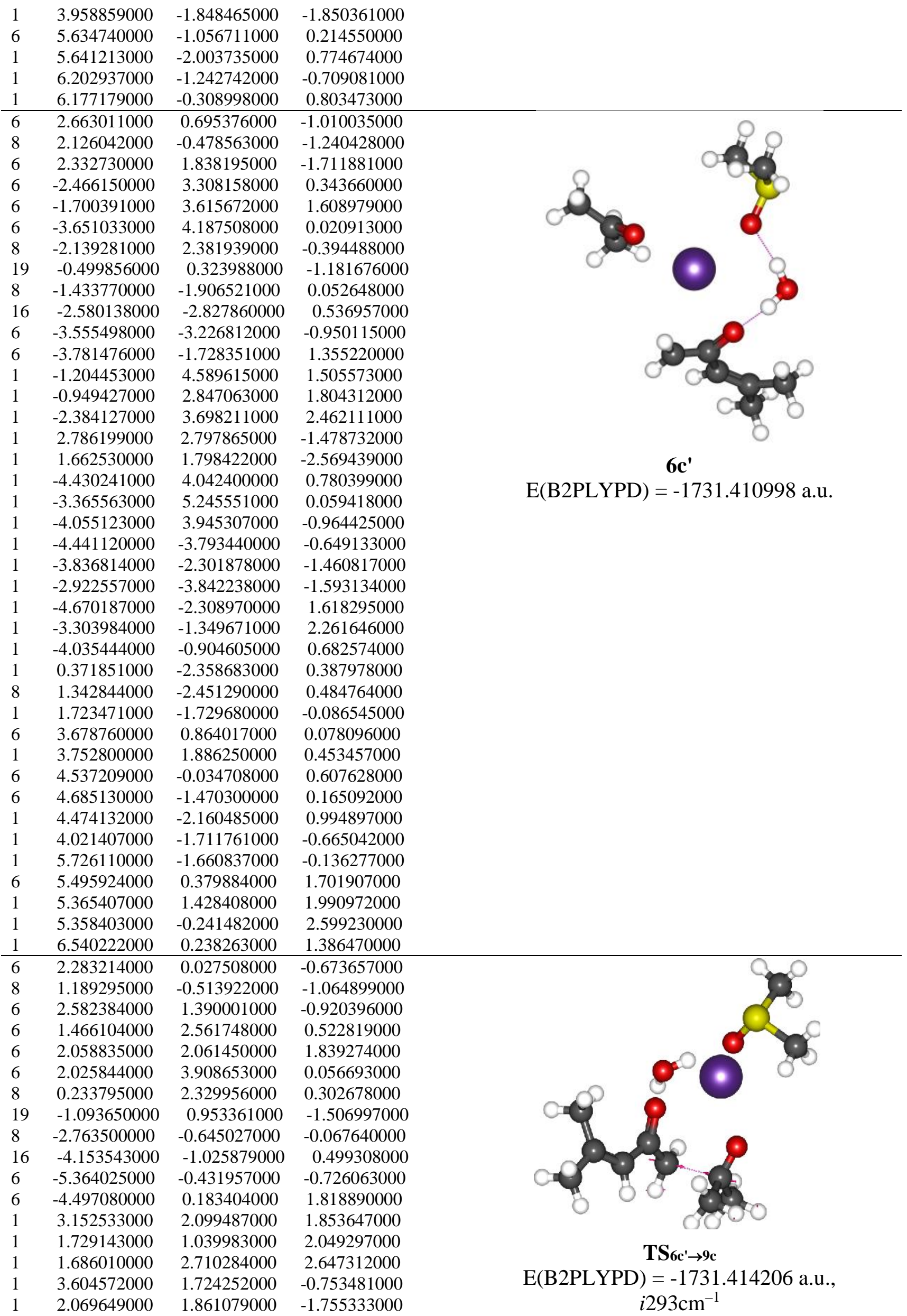




\begin{tabular}{|c|c|c|}
\hline 1.6 & 00 & .732243000 \\
\hline 3.118816000 & 3.951961000 & 0.088154000 \\
\hline 1.682497000 & 4.141854000 & -0.955824000 \\
\hline-6.370906000 & -0.583354000 & -0.3270 \\
\hline-5.181139000 & 0.625228000 & -0.93685000 \\
\hline-5.228854000 & -1.032090000 & -1.62851400 \\
\hline-5.517547000 & 0.029691000 & 2.18137300 \\
\hline-3.784399000 & -0.008938000 & 2.62389900 \\
\hline-4.367806000 & 1.195737000 & 1.42654400 \\
\hline-1.302903000 & -1.788832000 & 0.22388200 \\
\hline-0.470541000 & -2.304132000 & 0.29044300 \\
\hline 0.210486000 & -1.737921000 & -0.14821500 \\
\hline 3.269037000 & -0.733366000 & 0.14162200 \\
\hline 3.928319000 & -0.106762000 & 0.7 \\
\hline 3.501340000 & -2.065583000 & 0.16810500 \\
\hline 2.785684000 & -3.096762000 & -0.667 \\
\hline 2.190641000 & -3.765423000 & -0.03004500 \\
\hline 2.118882000 & -2.645856000 & -1.40292000 \\
\hline 3.520273000 & 882000 & -1.18292500 \\
\hline 4.590434000 & -2.626629000 & 1.05257500 \\
\hline 5.075294000 & 15000 & 1.6553 \\
\hline 4.182376000 & 4000 & 1.73 \\
\hline 5.361245000 & -3.130762000 & 0.45218400 \\
\hline-2.347072000 & 0.24 & -0.69545800 \\
\hline-1.340199000 & 479000 & -1.19808000 \\
\hline-2.610325000 & 100000 & \\
\hline-1.811 & 380000 & 0.11 \\
\hline-2.215099000 & -1.862870000 & 1.5780 \\
\hline-2.300226000 & 48000 & -0.212 \\
\hline-0.455708000 & -2.072434000 & -0.07 \\
\hline 1.022841000 & -0.5 & \\
\hline 3.012 & 1000 & 0.222 \\
\hline 4.171276000 & 38000 & 0.7716 \\
\hline 3.860 & 9000 & $0.16^{\circ}$ \\
\hline 5.615 & 30000 & -0.27170000 \\
\hline-3.300959000 & -1.910264000 & 1.73 \\
\hline-1.866 & 8000 & 1.86436200 \\
\hline-1.740552000 & 37000 & 2.25272400 \\
\hline-3.684770000 & -1.431396000 & -0.8009230 \\
\hline-2.280688000 & 16000 & -1.8942920 \\
\hline-1.785018000 & -4.338740000 & 0.44221800 \\
\hline-3.38 & 1000 & -0.07480800 \\
\hline-2.051561000 & 463000 & -1.25157300 \\
\hline 4.710031000 & 3.02 & 0.438568000 \\
\hline 70000 & 9000 & -0.9169 \\
\hline 2.956669000 & 329000 & 0.66493100 \\
\hline 6.436246000 & 3000 & -0.00101100 \\
\hline 5.897142000 & -0.718419000 & -0.06229600 \\
\hline 5.344693000 & 81000 & -1.324 \\
\hline 2.054533000 & -1.397970000 & 1.36736700 \\
\hline 1.446440000 & -2.042437000 & 1.78336400 \\
\hline 0.657280000 & 2000 & 1.1491 \\
\hline-3.323820000 & 487000 & 0.12046700 \\
\hline-4.06 & 0.371766000 & 0.62678600 \\
\hline-3.432599000 & 2.335565000 & 0.27266900 \\
\hline-4.520826000 & 2.903482000 & 1.14767200 \\
\hline-5.205905000 & 3.523181000 & 0.55262400 \\
\hline-5.102412000 & 2.124661000 & 1.64969800 \\
\hline-4.089361000 & 3.565759000 & 1.91105500 \\
\hline-2.542703000 & 3.362633000 & -0.37544400 \\
\hline-1.776277000 & 3.693525000 & 0.34107700 \\
\hline-2.024686000 & 2.972693000 & -1.2514880 \\
\hline
\end{tabular}

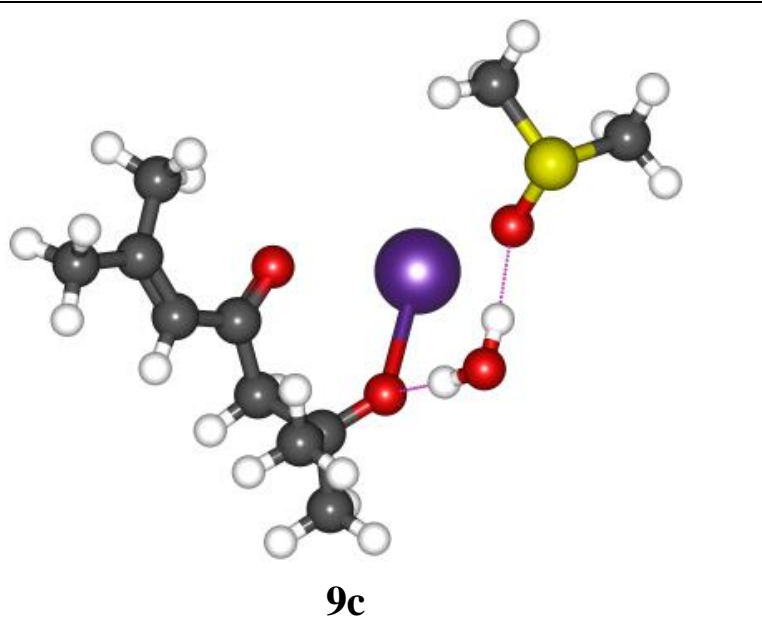

$\mathrm{E}(\mathrm{B} 2 \mathrm{PLYPD})=-1731.428017$ a.u. 


\begin{tabular}{|c|c|c|c|c|}
\hline 1 & -3.124764000 & 4.251980000 & -0.64642600 & \\
\hline 6 & -2.161853000 & 0.609263000 & -0.493946000 & \multirow{40}{*}{$\mathrm{E}(\mathrm{B} 2 \mathrm{PLYPD})=-1731.438476$ a.u. } \\
\hline 8 & -0.927736000 & 0.707496000 & -0.412801000 & \\
\hline 6 & -2.794631000 & -0.710710000 & -0.899529000 & \\
\hline 6 & -2.960681000 & -1.756868000 & 0.262839000 & \\
\hline 6 & -3.762932000 & -1.174699000 & 1.434128000 & \\
\hline 6 & -3.693393000 & -2.984316000 & -0.313077000 & \\
\hline 8 & -1.695270000 & -2.138732000 & 0.781185000 & \\
\hline 19 & 1.065279000 & -1.127516000 & 0.030457000 & \\
\hline 8 & 3.542429000 & -0.120989000 & 0.192107000 & \\
\hline 16 & 4.965438000 & 0.135649000 & -0.344270000 & \\
\hline 6 & 6.103149000 & -0.292938000 & 1.016626000 & \\
\hline 6 & 5.201418000 & 1.944726000 & -0.305223000 & \\
\hline 1 & -4.747064000 & -0.817482000 & 1.109010000 & \\
\hline 1 & -3.228510000 & -0.340327000 & 1.901843000 & \\
\hline 1 & -3.913555000 & -1.948020000 & 2.196305000 & \\
\hline 1 & -3.779043000 & -0.528807000 & -1.345983000 & \\
\hline 1 & -2.152762000 & -1.169511000 & -1.659305000 & \\
\hline 1 & -3.791916000 & -3.752009000 & 0.463616000 & \\
\hline 1 & -4.697253000 & -2.722358000 & -0.668728000 & \\
\hline 1 & -3.132748000 & -3.414118000 & -1.151262000 & \\
\hline 1 & 7.122563000 & -0.024063000 & 0.724907000 & \\
\hline 1 & 5.801491000 & 0.239350000 & 1.923145000 & \\
\hline 1 & 6.033150000 & -1.372604000 & 1.167008000 & \\
\hline 1 & 6.238455000 & 2.173221000 & -0.567599000 & \\
\hline 1 & 4.527305000 & 2.373967000 & -1.049862000 & \\
\hline 1 & 4.957791000 & 2.320716000 & 0.692446000 & \\
\hline 1 & -0.353941000 & -4.169100000 & -1.132992000 & \\
\hline 8 & -0.057617000 & -3.302908000 & -0.815736000 & \\
\hline 1 & -1.141611000 & -2.675145000 & 0.082266000 & \\
\hline 6 & -3.079610000 & 1.726832000 & -0.214590000 & \\
\hline 1 & -4.133566000 & 1.458742000 & -0.186049000 & \\
\hline 6 & -2.761738000 & 3.034367000 & -0.030979000 & \\
\hline 6 & -1.369413000 & 3.605186000 & -0.073683000 & \\
\hline 1 & -0.952128000 & 3.638838000 & 0.943492000 & \\
\hline 1 & -0.690293000 & 3.004933000 & -0.679821000 & \\
\hline 1 & -1.392281000 & 4.636924000 & -0.443017000 & \\
\hline 6 & -3.852203000 & 4.037869000 & 0.238535000 & \\
\hline 1 & -3.636551000 & 4.593042000 & 1.161908000 & \\
\hline 1 & -3.890620000 & 4.783262000 & -0.567768000 & \\
\hline 1 & -4.837365000 & 3.571780000 & 0.331416000 & \\
\hline 6 & 2.295026000 & 0.388221000 & -0.156079000 & \\
\hline 8 & 1.200664000 & 0.587481000 & -0.757674000 & \\
\hline 6 & 2.735547000 & -0.918531000 & 0.281712000 & \\
\hline 6 & 2.339955000 & -2.217046000 & -0.453636000 & \\
\hline 6 & 2.339107000 & -2.093320000 & -1.981894000 & \\
\hline 6 & 3.304982000 & -3.337529000 & -0.024480000 & \\
\hline 8 & 0.986274000 & -2.637142000 & -0.096249000 & \\
\hline 19 & -0.931412000 & -0.503462000 & 0.514680000 & \\
\hline 8 & -3.516742000 & 0.034764000 & 0.272963000 & \\
\hline 16 & -5.056714000 & 0.089167000 & 0.333407000 & \\
\hline 6 & -5.645965000 & -0.461409000 & -1.303406000 & \\
\hline 6 & -5.503245000 & 1.851748000 & 0.183307000 & \\
\hline 1 & 3.346595000 & -1.855747000 & -2.343356000 & \\
\hline 1 & 1.658324000 & -1.302497000 & -2.306306000 & \\
\hline 1 & 2.025762000 & -3.040959000 & -2.438807000 & \multirow{7}{*}{$\begin{array}{c}\text { TS } \mathbf{1 0 c \rightarrow 1 1 c} \\
\mathrm{E}(\mathrm{B} 2 \mathrm{PLYPD})=-1731.424408 \text { a.u. } \\
i 1365 \mathrm{~cm}^{-1}\end{array}$} \\
\hline 1 & 3.781747000 & -0.930945000 & 0.597945000 & \\
\hline 1 & 1.987063000 & -1.042952000 & 1.458299000 & \\
\hline 1 & 3.004138000 & -4.288888000 & -0.478937000 & \\
\hline 1 & 4.333640000 & -3.115825000 & -0.334421000 & \\
\hline 1 & 3.298548000 & -3.459126000 & 1.066137000 & \\
\hline 1 & -6.731884000 & -0.338737000 & -1.349320000 & \\
\hline
\end{tabular}




\begin{tabular}{|c|c|c|}
\hline-5.151636000 & 0.124338000 & -2.083516000 \\
\hline-5.388261000 & -1.518600000 & -1.398701000 \\
\hline-6.590524000 & 1.937659000 & 0.102106000 \\
\hline-5.159067000 & 2.349501000 & 1.092719000 \\
\hline-5.009763000 & 2.277094000 & -0.695075000 \\
\hline 1.519617000 & -1.643140000 & 3.089006000 \\
\hline 1.082146000 & -1.336360000 & 2.278176000 \\
\hline 0.925925000 & -2.523354000 & 0.881637000 \\
\hline 3.173893000 & 1.522064000 & 0.261244000 \\
\hline 4.004721000 & 1.225219000 & 0.901010000 \\
\hline 3.103777000 & 2.832335000 & -0.070768000 \\
\hline 2.067313000 & 3.461348000 & -0.967196000 \\
\hline 1.375003000 & 2.724522000 & -1.371572000 \\
\hline 1.496174000 & 4.217588000 & -0.408432000 \\
\hline 2.559876000 & 3.997975000 & -1.790939000 \\
\hline 4.138437000 & 3.798566000 & 0.457363000 \\
\hline 4.867595000 & 3.310289000 & 1.111835000 \\
\hline 4.681829000 & 4.276389000 & -0.370204000 \\
\hline 3.657119000 & 4.610687000 & 1.020708000 \\
\hline 2.457277000 & 0.224309000 & -0.273633000 \\
\hline 1.395265000 & 0.508710000 & -0.952189000 \\
\hline 2.823649000 & -1.061118000 & 0.145994000 \\
\hline 2.188655000 & -2.369885000 & -0.284776000 \\
\hline 1.994869000 & -2.486490000 & -1.803881000 \\
\hline 3.037316000 & -3.547770000 & 0.223197000 \\
\hline 0.825540000 & -2.544334000 & 0.261611000 \\
\hline-0.906183000 & -0.307121000 & 0.087262000 \\
\hline-3.547700000 & 0.046136000 & -0.022939000 \\
\hline-5.006941000 & 0.253121000 & 0.431220000 \\
\hline-6.047324000 & -0.504575000 & -0.862135000 \\
\hline-5.401782000 & 2.000106000 & 0.081631000 \\
\hline 2.969009000 & -2.486905000 & -2.306079000 \\
\hline 1.411636000 & -1.641700000 & -2.177823000 \\
\hline 1.477800000 & -3.422289000 & -2.053935000 \\
\hline 3.766937000 & -1.157790000 & 0.682192000 \\
\hline 1.666932000 & -0.563323000 & 1.867786000 \\
\hline 2.570069000 & -4.501028000 & -0.049936000 \\
\hline 4.045449000 & -3.519441000 & -0.208075000 \\
\hline 3.137502000 & -3.512355000 & 1.315856000 \\
\hline-7.097801000 & -0.289732000 & -0.646108000 \\
\hline-5.758291000 & -0.106776000 & -1.839022000 \\
\hline-5.874234000 & -1.582396000 & -0.823080000 \\
\hline-6.460675000 & 2.174493000 & 0.293231000 \\
\hline-4.786022000 & 2.608014000 & 0.748394000 \\
\hline-5.168823000 & 2.223531000 & -0.963278000 \\
\hline 1.135987000 & -0.468420000 & 3.342206000 \\
\hline 0.854024000 & -0.646073000 & 2.430769000 \\
\hline 0.872780000 & -2.362398000 & 1.218415000 \\
\hline 3.394052000 & 1.312454000 & 0.165038000 \\
\hline 4.209335000 & 0.958481000 & 0.798387000 \\
\hline 3.395231000 & 2.634850000 & -0.118490000 \\
\hline 2.389755000 & 3.355178000 & -0.984614000 \\
\hline 1.635920000 & 2.671066000 & -1.373456000 \\
\hline 1.894040000 & 4.153770000 & -0.411518000 \\
\hline 2.899221000 & 3.854188000 & -1.823087000 \\
\hline 4.481439000 & 3.527961000 & 0.440294000 \\
\hline 5.193838000 & 2.975646000 & 1.062759000 \\
\hline 5.042128000 & 4.018138000 & -0.369488000 \\
\hline 0 & 0 & 1.0 \\
\hline
\end{tabular}




\begin{tabular}{|c|c|c|c|}
\hline 6 & 2.487124000 & 0.319424000 & -0.338530000 \\
\hline 8 & 1.507901000 & 0.559577000 & -1.111050000 \\
\hline 6 & .913128000 & -0.991604000 & 0.043064000 \\
\hline 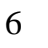 & 2.461635000 & -2.227893000 & -0.453678000 \\
\hline 6 & 1.891076000 & -2.370152000 & -1.847241000 \\
\hline 6 & 3.217745000 & -3.451259000 & 0.025031000 \\
\hline 8 & 0.677070000 & -2.609496000 & 0.512995000 \\
\hline 19 & -0.815339000 & -0.426730000 & -0.030592000 \\
\hline 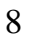 & -3.383400000 & 0.256743000 & -0.144721000 \\
\hline 16 & -4.867296000 & 0.356547000 & -0.552208000 \\
\hline 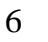 & -5.465939000 & 1.947027000 & 0.111774000 \\
\hline 6 & -5.777894000 & -0.746398000 & 0.581217000 \\
\hline 1 & 2.726865000 & -2.360122000 & -2.564448000 \\
\hline 1 & 1.225746000 & -1.547244000 & -2.102901000 \\
\hline 1 & 41000 & 23000 & -1.962230000 \\
\hline 1 & 3.634943000 & 3000 & 7137000 \\
\hline 1 & 0.702287000 & & 0000 \\
\hline 1 & 742000 & -4.3 & -0.154169000 \\
\hline 1 & 37000 & 5000 & 0000 \\
\hline 1 & 3.46 & 3000 & 12000 \\
\hline 1 & 370000 & 000 & 5000 \\
\hline 1 & -5.2 & & 4000 \\
\hline 1 & -4.9 & & 8000 \\
\hline 1 & -6.8 & -0.6 & 6000 \\
\hline 1 & & & \\
\hline 1 & 502000 & -0 & 000 \\
\hline 1 & 1.43 & -0.5 & 5000 \\
\hline 8 & 0.6 & & 2000 \\
\hline 1 & 00 & -3 & 4000 \\
\hline 6 & & & \\
\hline 1 & 4.0 & & 00 \\
\hline 6 & 000 & 000 & 000 \\
\hline 6 & 2.24 & 000 & 4000 \\
\hline 1 & 1.6 & 00 & 1000 \\
\hline 1 & 372000 & & 3000 \\
\hline 1 & 2.8 & & 000 \\
\hline 6 & 4.1 & 3.6 & 00 \\
\hline 1 & 4.80 & & \\
\hline 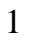 & 4.736292 & 4.2 & 1000 \\
\hline 1 & 3.559303000 & 64000 & \\
\hline 6 & & & \\
\hline 8 & 1.5 & 00 & 3000 \\
\hline 6 & 3.92 & 000 & 1000 \\
\hline 6 & 4.208918000 & 9000 & -0.631178000 \\
\hline 6 & 3.203998000 & -1.9 & 33000 \\
\hline 6 & 5.63 & -1.4 & 9000 \\
\hline . & -0.651488000 & -3.924 & 282000 \\
\hline 19 & & & 000 \\
\hline . & -2.997991000 & -0.127146000 & -0.342382000 \\
\hline 16 & -4.319790000 & 0.464791000 & 9953000 \\
\hline 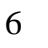 & 616000 & 1.8 & 0.234452000 \\
\hline 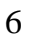 & -5.638110000 & -0.674233000 & -0.328269000 \\
\hline 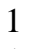 & 54000 & 26000 & -2.016241000 \\
\hline 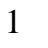 & 2.346384000 & -1.410846000 & -1.666606000 \\
\hline 1 & 2.816272000 & -2.623084000 & -0.478368000 \\
\hline 1 & & & \\
\hline 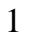 & -0.452120000 & -3.548430000 & 1.569831000 \\
\hline 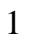 & & -2.478244000 & -0.162085000 \\
\hline 1 & 5.980237000 & -1.629357000 & -1.675056000 \\
\hline 1 & 6.316873000 & -0.809807000 & -0.129988000 \\
\hline 1 & -5.711681000 & 2.248815000 & -0.030921000 \\
\hline 1 & -4.705008000 & 1.515959000 & 1.272633000 \\
\hline
\end{tabular}
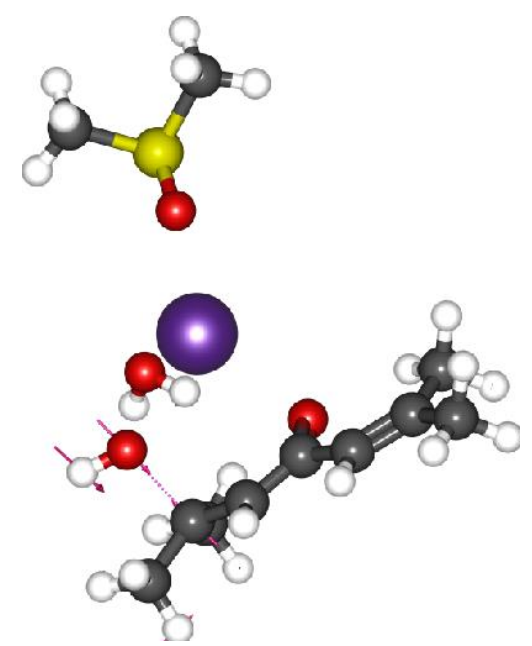

TS11c $\rightarrow$ 12c'

$\mathrm{E}(\mathrm{B} 2 \mathrm{PLYPD})=-1731.418612$ a.u., $i 284 \mathrm{~cm}^{-1}$ 


$\begin{array}{rrr}-3.966460000 & 2.628787000 & 0.072594000 \\ -6.608933000 & -0.241747000 & -0.586799000 \\ -5.494878000 & -1.613067000 & -0.867857000 \\ -5.556531000 & -0.837347000 & 0.750005000 \\ 0.534229000 & -3.284027000 & 2.818090000 \\ -0.359958000 & -3.083752000 & 2.501969000 \\ -0.744339000 & -4.883364000 & 0.008948000 \\ 2.647251000 & 2.289984000 & 0.391337000 \\ 3.627026000 & 2.647308000 & 0.702106000 \\ 1.637611000 & 3.196429000 & 0.365233000 \\ 0.224110000 & 2.919432000 & -0.071757000 \\ 0.144205000 & 2.030300000 & -0.696446000 \\ -0.412120000 & 2.762836000 & 0.811805000 \\ -0.184025000 & 3.788170000 & -0.602364000 \\ 1.899208000 & 4.616903000 & 0.795425000 \\ 2.916811000 & 4.759993000 & 1.170466000 \\ 1.735016000 & 5.306340000 & -0.044186000 \\ 1.190206000 & 4.912598000 & 1.581015000 \\ -0.000001000 & 0.160512000 & -0.188312000 \\ -0.000006000 & 1.398127000 & -0.314687000 \\ 1.242392000 & -0.643747000 & -0.116817000 \\ 2.516173000 & -0.203307000 & 0.038362000 \\ 2.934487000 & 1.236230000 & 0.178456000 \\ 3.649883000 & -1.196180000 & 0.084791000 \\ 3.736685000 & 1.324450000 & 0.921755000 \\ 2.104332000 & 1.889768000 & 0.443446000 \\ 3.351166000 & 1.594006000 & -0.774901000 \\ 1.094690000 & -1.720473000 & -0.176833000 \\ 4.405980000 & -0.946459000 & -0.672379000 \\ 4.159276000 & -1.149694000 & 1.057233000 \\ 3.313475000 & -2.223508000 & -0.083378000 \\ -1.242382000 & -0.643751000 & -0.116737000 \\ -1.094661000 & -1.720484000 & -0.176576000 \\ -2.516179000 & -0.203310000 & 0.038351000 \\ -2.934515000 & 1.236240000 & 0.178198000 \\ -2.104396000 & 1.889814000 & 0.443221000 \\ -3.351046000 & 1.593883000 & -0.775276000 \\ -3.736818000 & 1.324565000 & 0.921371000 \\ -3.649858000 & -1.196208000 & 0.084978000 \\ -3.313431000 & -2.223551000 & -0.083054000 \\ -4.159209000 & -1.149591000 & 1.057436000 \\ -4.405996000 & -0.946613000 & -0.672192000\end{array}$

Figure 5

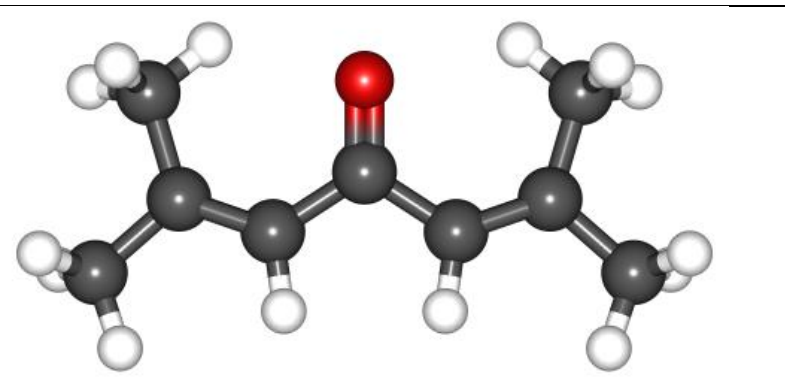

12c

$\mathrm{E}(\mathrm{B} 2 \mathrm{PLYPD})=-426.3191873$ a.u.

$\begin{array}{llll}6 & 0.000000000 & 0.000000000 & -0.604642000 \\ 1 & 0.000000000 & 0.000000000 & -1.674234000 \\ 6 & 0.000000000 & 0.000000000 & 0.604685000 \\ 1 & 0.000000000 & 0.000000000 & 1.673974000\end{array}$




\begin{tabular}{|c|c|c|c|c|}
\hline 6 & 3.274639000 & 1.838622000 & 0.012898000 & \\
\hline 16 & 3.355432000 & 0.055773000 & -0.367182000 & \\
\hline 6 & 4.250771000 & -0.482805000 & 1.128715000 & \\
\hline 8 & 1.916574000 & -0.478149000 & -0.215694000 & \\
\hline 19 & -0.589249000 & -1.176527000 & -0.717426000 & \\
\hline 8 & -2.389754000 & -1.946459000 & 1.062680000 & \\
\hline 6 & -4.027032000 & -0.210570000 & -0.088752000 & \\
\hline 6 & -3.183551000 & 0.954135000 & -0.146087000 & \\
\hline 6 & -3.373662000 & 2.045779000 & 0.899417000 & \\
\hline 8 & -2.235301000 & 1.074337000 & -0.966546000 & \\
\hline 1 & -2.856270000 & -2.457762000 & 1.743073000 & \\
\hline 1 & 5.249549000 & -0.036832000 & 1.125928000 & \\
\hline 1 & 3.688657000 & -0.180291000 & 2.016713000 & \\
\hline 1 & 4.330976000 & -1.571138000 & 1.080388000 & $\mathbf{T S}_{1 \rightarrow 2}$ \\
\hline 1 & 4.289000000 & 2.247665000 & 0.014313000 & $\mathrm{E}(\mathrm{B} 2 \mathrm{PLYPD})=-1421.713615$ a.u. \\
\hline 1 & 2.686728000 & 2.308689000 & -0.778711000 & $I 1291 \mathrm{~cm}^{-1}$ \\
\hline 1 & 2.792205000 & 1.982410000 & 0.983729000 & \\
\hline 1 & -3.928423000 & 1.693174000 & 1.774592000 & \\
\hline 1 & -2.404527000 & 2.447453000 & 1.213673000 & \\
\hline 1 & -3.945246000 & 2.869662000 & 0.450267000 & \\
\hline 1 & -4.159744000 & -0.716926000 & -1.049796000 & \\
\hline 1 & -4.960838000 & -0.093889000 & 0.466916000 & \\
\hline 1 & -3.252399000 & -1.099355000 & 0.584253000 & \\
\hline 6 & 3.036683000 & 3.676657000 & -0.274442000 & \\
\hline 6 & 2.751751000 & 2.506173000 & -0.143230000 & \\
\hline 6 & 3.892939000 & -1.018007000 & 1.453055000 & \\
\hline 6 & 3.183237000 & -1.065384000 & 0.284891000 & \\
\hline 6 & 3.711066000 & -1.867372000 & -0.897713000 & \\
\hline 8 & 2.037738000 & -0.448664000 & 0.072690000 & \\
\hline 19 & 0.009477000 & -0.455838000 & -1.620574000 & \\
\hline 8 & -1.887986000 & -0.072069000 & 0.284513000 & \\
\hline 16 & -3.375764000 & 0.121089000 & 0.668130000 & \\
\hline 6 & -4.039025000 & 1.337694000 & -0.516003000 & \\
\hline 6 & -4.242907000 & -1.355115000 & 0.042921000 & \\
\hline 8 & 0.306328000 & -0.001328000 & 2.133410000 & \\
\hline 1 & 1.014227000 & -0.154925000 & 1.446750000 & $2^{\prime \prime}$ \\
\hline 1 & -5.116964000 & 1.432448000 & -0.356937000 & $\mathrm{E}(\mathrm{B} 2 \mathrm{PLYPD})=-1498.984020$ a.u. \\
\hline 1 & -3.548116000 & 2.290601000 & -0.306385000 & \\
\hline 1 & -3.821161000 & 1.009952000 & -1.536130000 & \\
\hline 1 & -5.318517000 & -1.219467000 & 0.187034000 & \\
\hline 1 & -4.003877000 & -1.496443000 & -1.014732000 & \\
\hline 1 & -3.894030000 & -2.206404000 & 0.631582000 & \\
\hline 1 & 4.833418000 & -1.550862000 & 1.563427000 & \\
\hline 1 & 3.530290000 & -0.449633000 & 2.307714000 & \\
\hline 1 & 2.489320000 & 1.456714000 & -0.032261000 & \\
\hline 1 & 3.290239000 & 4.709314000 & -0.387719000 & \\
\hline 1 & -0.526745000 & -0.002907000 & 1.616741000 & \\
\hline 1 & 2.982648000 & -2.637736000 & -1.186481000 & \\
\hline 1 & 3.842733000 & -1.211197000 & -1.769088000 & \\
\hline 1 & 4.667597000 & -2.357330000 & -0.684454000 & \\
\hline 6 & 5.260562000 & -1.681437000 & 1.362542000 & \\
\hline 6 & 4.126207000 & -1.345174000 & 0.907453000 & \\
\hline 6 & 3.798271000 & -0.499351000 & -1.031193000 & \\
\hline 6 & 2.979063000 & 0.569915000 & -0.652421000 & \\
\hline 6 & 3.593143000 & 1.934621000 & -0.386217000 & \\
\hline 8 & 1.724713000 & 0.437641000 & -0.392658000 & \\
\hline 19 & -0.377724000 & 1.603211000 & 0.746481000 & \\
\hline 8 & -2.138000000 & -0.261837000 & -0.139401000 & \\
\hline 16 & -3.595125000 & -0.784459000 & -0.198376000 & \\
\hline 6 & -4.245063000 & -0.624642000 & 1.496574000 & \\
\hline 6 & -4.565582000 & 0.545576000 & -0.979919000 & \\
\hline 8 & 0.114684000 & -1.655012000 & -1.212200000 & \\
\hline
\end{tabular}


0.783224000

$-5.309267000$

$-3.697976000$

$-4.086390000$

$-5.623834000$

$-4.392695000$

$-4.226610000$

4.817977000

3.334096000

3.075702000

6.275093000

$-0.735751000$

3.117994000

3.404020000

4.672398000
$-0.970563000$

$-0.877096000$

$-1.336077000$

0.397411000

0.270378000

1.484301000

0.624532000

$-0.307146000$

$-1.401890000$

$-1.356347000$

$-1.592573000$

$-1.284778000$

2.686676000

2.235590000

1.954533000
$-0.950393000$

1.491867000

2.119004000

1.851543000

$-0.955614000$

$-0.446579000$

$-2.015171000$

$-1.349980000$

$-1.422847000$

1.162335000

0.996268000

$-0.892419000$

$-1.029784000$

0.652990000

$-0.567193000$

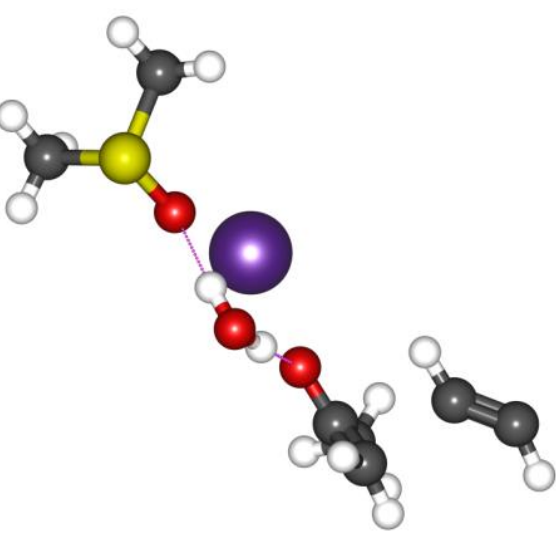

$\mathbf{T S}_{2}{ }^{\prime \prime} \rightarrow \mathbf{1 8}^{\prime}$

$\mathrm{E}(\mathrm{B} 2 \mathrm{PLYPD})=-1498.9582709$ a.u., $i 455 \mathrm{~cm}^{-1}$

\begin{tabular}{lc}
\hline 6 & 5.417235000 \\
6 & 4.226722000 \\
6 & 3.878098000 \\
6 & 3.006409000 \\
6 & 3.678092000 \\
8 & 1.775740000 \\
19 & -0.494162000 \\
8 & -2.157283000 \\
16 & -3.602497000 \\
6 & -4.301658000 \\
6 & -4.575282000 \\
8 & 0.058523000 \\
1 & 0.756213000 \\
1 & -5.359906000 \\
1 & -3.759255000 \\
1 & -4.173902000 \\
1 & -5.629218000 \\
1 & -4.429533000 \\
1 & -4.213999000 \\
1 & 4.801438000 \\
1 & 3.332149000 \\
1 & 3.343800000 \\
1 & 6.170675000 \\
1 & -0.773058000 \\
1 & 3.932599000 \\
1 & 3.020281000 \\
1 & 4.617192000 \\
\hline 6 & -2.466327000 \\
6 & -1.434902000 \\
6 & -0.296901000 \\
6 & 1.024433000 \\
6 & 1.468296000 \\
8 & 1.674840000 \\
1 & -0.482104000 \\
1 & -0.207238000 \\
1 & -1.376247000 \\
1 & -2.561600000 \\
1 & 2.315331000 \\
1 & 0.639436000 \\
1 & 1.762378000 \\
1 & -3.256275000 \\
& \\
1
\end{tabular}

$-1.372230000$

$-1.363333000$

$-0.688107000$

0.494643000

1.829260000

0.379368000

1.816953000

$-0.275730000$

$-0.836923000$

$-0.321060000$

0.258106000

$-1.887532000$

$-1.210818000$

$-0.596159000$

$-0.863482000$

0.757819000

$-0.026103000$

1.297408000

0.104334000

$-0.375638000$

$-1.386784000$

$-1.858420000$

$-0.794991000$

$-1.418343000$

2.252156000

2.527498000

1.707664000

0.390991000

$-0.457973000$

$-0.364977000$

$-0.136688000$

1.293447000

$-1.083672000$

0.448401000

$-1.307628000$

$-1.286528000$

1.229753000

1.348747000

1.907864000

1.711041000

0.268920000
1.245889000

0.609494000

$-0.750338000$

$-0.455964000$

$-0.243234000$

$-0.335054000$

0.349157000

$-0.073812000$

$-0.045913000$

1.555222000

$-1.129337000$

$-0.795665000$

$-0.667594000$

1.579843000

2.332687000

1.678953000

$-1.062199000$

$-0.822762000$

$-2.148497000$

$-1.248223000$

$-1.396926000$

1.035169000

0.665490000

$-0.561603000$

$-1.225148000$

0.280562000

0.306740000

$-0.247640000$

$-0.188212000$

0.804418000

0.068563000

$-0.123657000$

$-0.357137000$

1.514144000

1.358105000

$-0.894425000$

0.439728000

$-0.811215000$

$-0.494177000$

0.848793000

$-0.984687000$

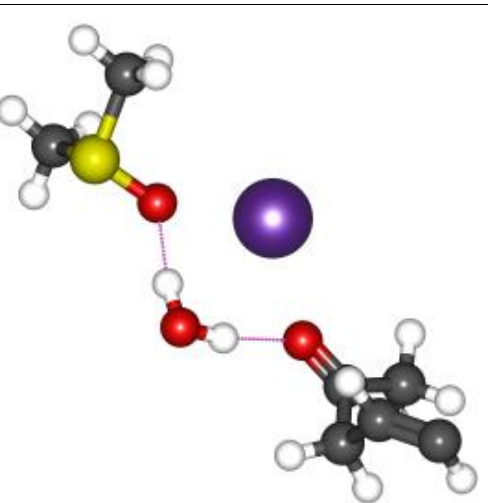

18

$\mathrm{E}(\mathrm{B} 2 \mathrm{PLYPD})=-1498.946819$ a.u.

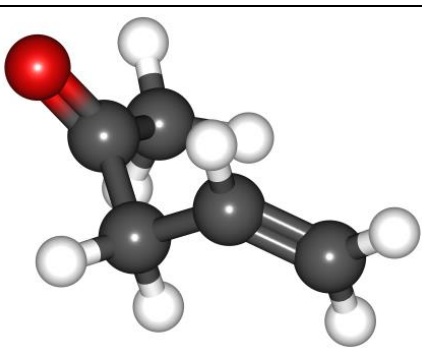

18

$\mathrm{E}(\mathrm{B} 2 \mathrm{PLYPD})=-1498.946819$ a.u. 


\begin{tabular}{|c|c|c|c|c|}
\hline 8 & 2.884638000 & 1.015033000 & 0.493645000 & \multirow{17}{*}{ 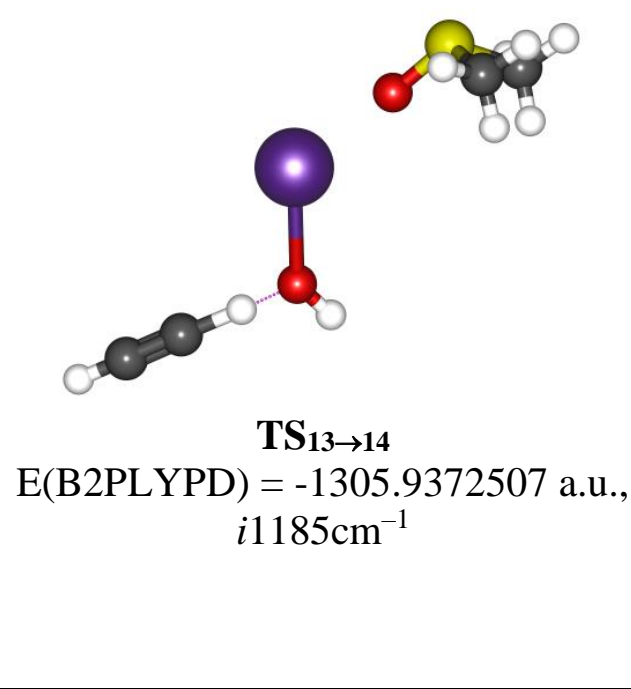 } \\
\hline 19 & 1.035503000 & -0.604250000 & -0.399176000 & \\
\hline 8 & -1.589092000 & -0.398230000 & -0.295317000 & \\
\hline 16 & -3.120548000 & -0.235932000 & -0.378271000 & \\
\hline 6 & -3.441161000 & 1.559282000 & -0.317452000 & \\
\hline 6 & -3.760815000 & -0.663623000 & 1.275830000 & \\
\hline 6 & 5.322027000 & 0.167143000 & 0.181781000 & \\
\hline 1 & -4.521071000 & 1.726099000 & -0.269659000 & \\
\hline 1 & -2.939091000 & 1.988497000 & 0.554047000 & \\
\hline 1 & -3.040545000 & 1.988370000 & -1.238655000 & \\
\hline 1 & -4.836171000 & -0.465422000 & 1.303468000 & \\
\hline 1 & -3.576907000 & -1.729944000 & 1.425300000 & \\
\hline 1 & -3.233701000 & -0.075080000 & 2.032081000 & \\
\hline 1 & 2.918151000 & 1.886053000 & 0.920224000 & \\
\hline 1 & 4.075489000 & 0.625384000 & 0.353985000 & \\
\hline 6 & 6.474290000 & -0.235261000 & 0.036671000 & \\
\hline 1 & 7.477636000 & -0.587970000 & -0.091694000 & \\
\hline 8 & 2.995816000 & 0.972402000 & 0.438306000 & \multirow{17}{*}{$\mathrm{E}(\mathrm{B} 2 \mathrm{PLYPD})=-1305.9273258 \mathrm{a} . \mathrm{u}$} \\
\hline 19 & 0.967955000 & -0.591644000 & -0.324837000 & \\
\hline 8 & -1.643109000 & -0.349684000 & -0.158387000 & \\
\hline 16 & -3.149075000 & -0.158804000 & -0.432460000 & \\
\hline 6 & -3.493113000 & 1.609337000 & -0.141475000 & \\
\hline 6 & -4.013954000 & -0.827947000 & 1.027764000 & \\
\hline 6 & 5.632494000 & 0.132910000 & 0.135609000 & \\
\hline 1 & -4.571742000 & 1.777489000 & -0.210335000 & \\
\hline 1 & -3.113544000 & 1.894770000 & 0.843723000 & \\
\hline 1 & -2.979670000 & 2.170034000 & -0.925814000 & \\
\hline 1 & -5.084241000 & -0.625661000 & 0.928758000 & \\
\hline 1 & -3.840014000 & -1.906290000 & 1.038472000 & \\
\hline 1 & -3.609719000 & -0.366321000 & 1.932961000 & \\
\hline 1 & 3.020093000 & 1.861174000 & 0.825562000 & \\
\hline 1 & 3.979268000 & 0.683612000 & 0.335734000 & \\
\hline 6 & 6.801197000 & -0.261849000 & 0.008859000 & \\
\hline 1 & 7.812233000 & -0.603147000 & -0.101707000 & \\
\hline 6 & 3.910146000 & -0.979514000 & 1.336471000 & \multirow{27}{*}{$\begin{array}{l}\mathbf{1 4}^{\prime} \\
=-1498.996485 \mathrm{a} . \mathrm{u} .\end{array}$} \\
\hline 16 & 3.126987000 & -1.068529000 & -0.306440000 & \\
\hline 6 & 3.753415000 & 0.501902000 & -0.985440000 & \\
\hline 8 & 1.613186000 & -0.843322000 & -0.059273000 & \\
\hline 19 & -0.222197000 & 0.986161000 & 0.839641000 & \\
\hline 8 & -2.396608000 & -0.716211000 & 0.101490000 & \\
\hline 6 & -3.626269000 & -0.709869000 & 0.025410000 & \\
\hline 6 & -0.215440000 & 3.660677000 & -0.318530000 & \\
\hline 6 & -0.220770000 & 4.825731000 & -0.756719000 & \\
\hline 6 & -4.378147000 & -1.838776000 & -0.633685000 & \\
\hline 6 & -4.438243000 & 0.429376000 & 0.587708000 & \\
\hline 8 & -0.411446000 & -2.670146000 & -0.757368000 & \\
\hline 1 & 3.466315000 & 1.325031000 & -0.325712000 & \\
\hline 1 & 3.303171000 & 0.626947000 & -1.972525000 & \\
\hline 1 & 4.841079000 & 0.435302000 & -1.078588000 & \\
\hline 1 & 3.596779000 & -0.059598000 & 1.837476000 & \\
\hline 1 & 3.578014000 & -1.854713000 & 1.899275000 & \\
\hline 1 & 4.996230000 & -1.008114000 & 1.211260000 & \\
\hline 1 & 0.371646000 & -2.109846000 & -0.554330000 & \\
\hline 1 & -1.173891000 & -2.106183000 & -0.517929000 & \\
\hline 1 & -4.990662000 & 0.073098000 & 1.467382000 & \\
\hline 1 & -3.795659000 & 1.262416000 & 0.879879000 & \\
\hline 1 & -5.185805000 & 0.767162000 & -0.139186000 & \\
\hline 1 & -5.230811000 & -2.148379000 & -0.018927000 & \\
\hline 1 & -3.722224000 & -2.691050000 & -0.823403000 & \\
\hline 1 & -4.788109000 & -1.482015000 & -1.588086000 & \\
\hline 1 & -0.225345000 & 5.829601000 & -1.136826000 & \\
\hline
\end{tabular}




\begin{tabular}{|c|c|c|c|}
\hline 6 & 4.349282000 & -0.420381000 & \\
\hline 16 & 3.298471000 & -0.411241000 & -0.469737000 \\
\hline 6 & 3.609232000 & 1.302537000 & -1.003965000 \\
\hline 0 & 1.838515000 & -0.432022000 & 0.048953000 \\
\hline 19 & -0.243738000 & 0.908064000 & 1.185426000 \\
\hline 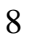 & -2.090900000 & -1.013419000 & 0.596734000 \\
\hline 6 & -3.123027000 & -0.531544000 & 0.056168000 \\
\hline 6 & -2.493540000 & 1.564668000 & -0.691469000 \\
\hline 6 & -1.960521000 & 2.617288000 & -1.070037000 \\
\hline 6 & -3.541839000 & 866000 & 3000 \\
\hline 6 & -4.240009000 & -0.028327000 & 55000 \\
\hline 8 & -0.027764000 & 7000 & -0.619218000 \\
\hline 1 & 3.356512000 & 1.989523000 & 9000 \\
\hline 1 & 2.975925000 & 1.487 & -1. \\
\hline 1 & 4.66 & 1.3 & 000 \\
\hline 1 & 9000 & 0.3 & 5000 \\
\hline 1 & 4.215892000 & -1.3 & 0000 \\
\hline 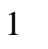 & 5.39 & -0.2 & 00 \\
\hline 1 & 09000 & -1.8 & 000 \\
\hline 1 & 50000 & -2.0 & 000 \\
\hline 1 & -4.7 & -0.9 & 000 \\
\hline 1 & -3.8 & 0.5 & \\
\hline 1 & -4.9 & & \\
\hline 1 & -4.00 & -2.0 & 000 \\
\hline 1 & & & \\
\hline 1 & -4.27 & -0.3 & 000 \\
\hline 1 & -1.515877000 & 3.53 & 00000 \\
\hline 6 & 4.41 & 0.1 & \\
\hline 16 & 5000 & 000 & 000 \\
\hline 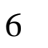 & 3.85 & -0.9 & 000 \\
\hline 8 & 1.92 & 0.30 & 000 \\
\hline 19 & -0.104086000 & 9000 & 71000 \\
\hline 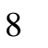 & -2.0 & & 000 \\
\hline 6 & 69000 & 0.2 & 000 \\
\hline 6 & -2.78 & -0.9 & \\
\hline 6 & -2.417113000 & -1.86 & 000 \\
\hline 6 & -3.683745000 & 1.37 & 000 \\
\hline 6 & 23000 & -0.2 & 3000 \\
\hline 8 & -0.179952000 & 7000 & 37000 \\
\hline 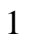 & 3.63 & -1.8 & \\
\hline 1 & 3.26 & -0.90 & 7000 \\
\hline 1 & 4.921567000 & -0.87 & 58000 \\
\hline 1 & & & \\
\hline . & 4.190604000 & 0.974 & 56000 \\
\hline 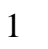 & 5.46 & 0.23 & 5000 \\
\hline 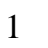 & 0.61 & 1.696 & 7000 \\
\hline 1 & -0.935276000 & 1000 & 06000 \\
\hline 1 & -4.57 & 0.58 & -1.58 \\
\hline 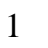 & -3.928523000 & -1.067 & -1.579654000 \\
\hline 1 & -5.168640000 & -0.58 & 35000 \\
\hline 1 & -3.964188000 & 2.214869000 & 0.255317000 \\
\hline 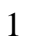 & -2.906321000 & 1.707370000 & 1.591235000 \\
\hline 1 & & & 1.478465000 \\
\hline 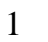 & -2.105284000 & -2.681425000 & 2.193755000 \\
\hline
\end{tabular}

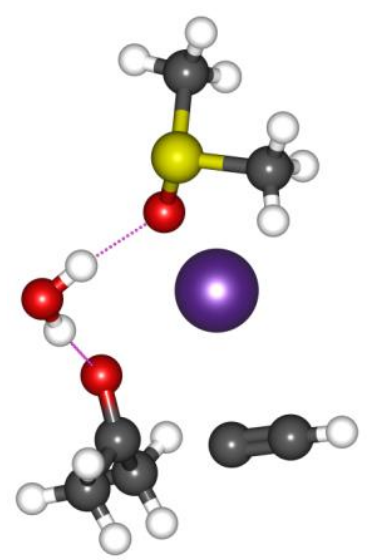

$\mathrm{TS}_{14} \rightarrow 15$

$\mathrm{E}(\mathrm{B} 2 \mathrm{PLYPD})=-1498.996662$ a.u., $i 203 \mathrm{~cm}^{-1}$ 


\begin{tabular}{|c|c|c|c|}
\hline 6 & 0.862220000 & -1.204441000 & -0.753886000 \\
\hline 6 & 0.310810000 & 0.002723000 & 0.025701000 \\
\hline 6 & -1.167291000 & 0.020987000 & 8000 \\
\hline 6 & -2.377773000 & 0.023386000 & -0.086145000 \\
\hline 8 & 0.730557000 & -0.066325000 & 1.408227000 \\
\hline 6 & 0.887775000 & 1.320490000 & -0.509390000 \\
\hline 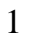 & 1.979812000 & 1.294222000 & -0.432285000 \\
\hline 1 & 0.511173000 & 2.167648000 & 61000 \\
\hline 1 & 0.611181000 & 1.461636000 & -1.558360000 \\
\hline 1 & 0.573298000 & -1.150212000 & -1.808739000 \\
\hline 1 & 0.477945000 & -2.144068000 & -0.341344000 \\
\hline 1 & 1.955212000 & -1.209507000 & -0.686293000 \\
\hline 1 & -3.446183000 & 0.028170000 & 7000 \\
\hline 1 & 0.398656000 & -0.896160000 & 357000 \\
\hline 6 & -3.425789000 & -3.614575000 & -0.582061000 \\
\hline 16 & -3.712685000 & -2.288917000 & 23000 \\
\hline 6 & -5.341679000 & -1.729658000 & 097000 \\
\hline 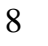 & -2.720035000 & 415000 & 27000 \\
\hline 19 & -1.284808000 & 0.314260000 & 53000 \\
\hline 0 & -1.103169000 & 0.266406000 & 16000 \\
\hline 1 & -1.703224000 & -0.280788000 & 23000 \\
\hline 1 & -6.066021000 & -2.536948000 & 8000 \\
\hline 1 & 03000 & 8000 & 6000 \\
\hline 1 & -5.266690000 & -1.450189000 & 9000 \\
\hline 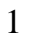 & -4.175586000 & -4.397281000 & 49000 \\
\hline 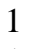 & 231000 & 5000 & 4000 \\
\hline 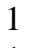 & -2.428463000 & -4.016395000 & 70000 \\
\hline 1 & 37000 & 2000 & 7000 \\
\hline 6 & 4.822777000 & 60000 & 9000 \\
\hline 6 & & & \\
\hline 6 & 14000 & -0.6 & 000 \\
\hline 6 & 2.638663000 & 98000 & 8000 \\
\hline 8 & 5000 & 3000 & 3000 \\
\hline 6 & 4.906490000 & -2.178617000 & 407000 \\
\hline 1 & 5.817095000 & -2.785623000 & 70000 \\
\hline 1 & 4.934102000 & -1.4 & 38000 \\
\hline 1 & 4.040715000 & -2.829878000 & 359000 \\
\hline 1 & 3.9435 & -3.16 & 70000 \\
\hline 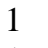 & 4.808925000 & -2.016065000 & 2000 \\
\hline 1 & 5.724856000 & -3.135672000 & 6000 \\
\hline 1 & 7000 & 97000 & 00000 \\
\hline 8 & 0.124648000 & 1.707912000 & 0.296260000 \\
\hline 6 & -0.0193890 & 3.08 & 3000 \\
\hline 6 & 1.152835000 & 3.725710000 & 5000 \\
\hline 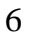 & -1.293538000 & 3.420828000 & 62000 \\
\hline 6 & -0.081483000 & 3.742584000 & 04000 \\
\hline 1 & 1.047669000 & 4.814055000 & -0.673054000 \\
\hline 1 & 1.210270000 & 3.290048000 & -1.580485000 \\
\hline 1 & 2.091643000 & 3.510035000 & -0.052400000 \\
\hline 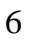 & -2.332078000 & 3.612509000 & -1.106573000 \\
\hline 1 & 0.849945000 & 3.515492000 & 2.156738000 \\
\hline 1 & -0.917822000 & 3.330228000 & 2.197924000 \\
\hline 1 & -0.197816000 & 4.832744000 & 1.569610000 \\
\hline 1 & -3.247411000 & 3.795022000 & -1.627740000 \\
\hline 1 & 1.759702000 & 0.728190000 & 0.189365000 \\
\hline
\end{tabular}

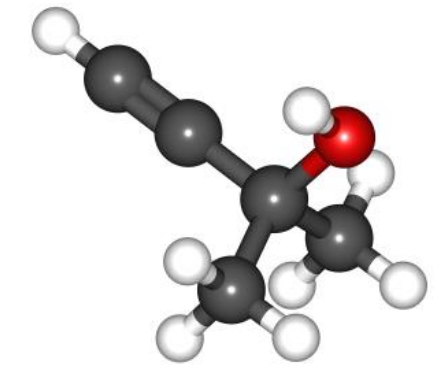

16

$\mathrm{E}(\mathrm{B} 2 \mathrm{PLYPD})=-270.3434979$ a.u.

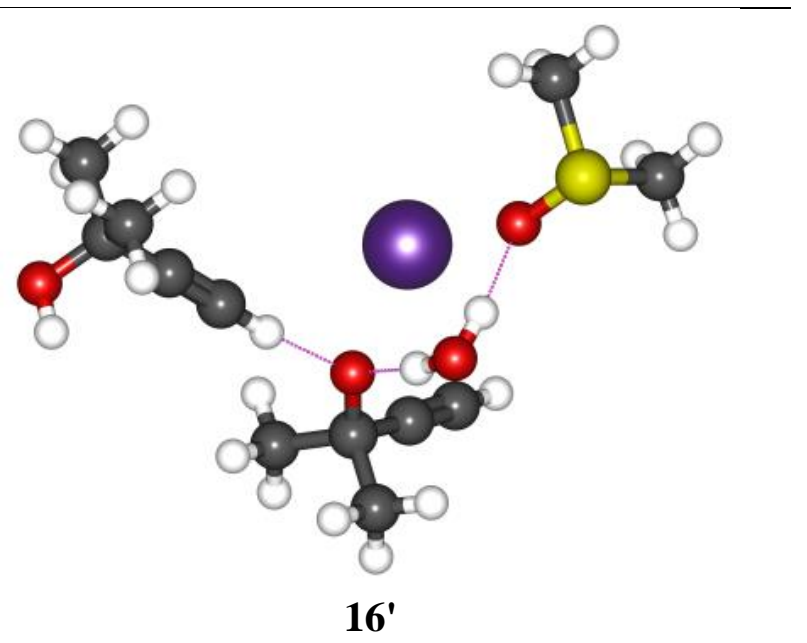

$\mathrm{E}(\mathrm{B} 2 \mathrm{PLYPD})=-1769.381469$ a.u. 


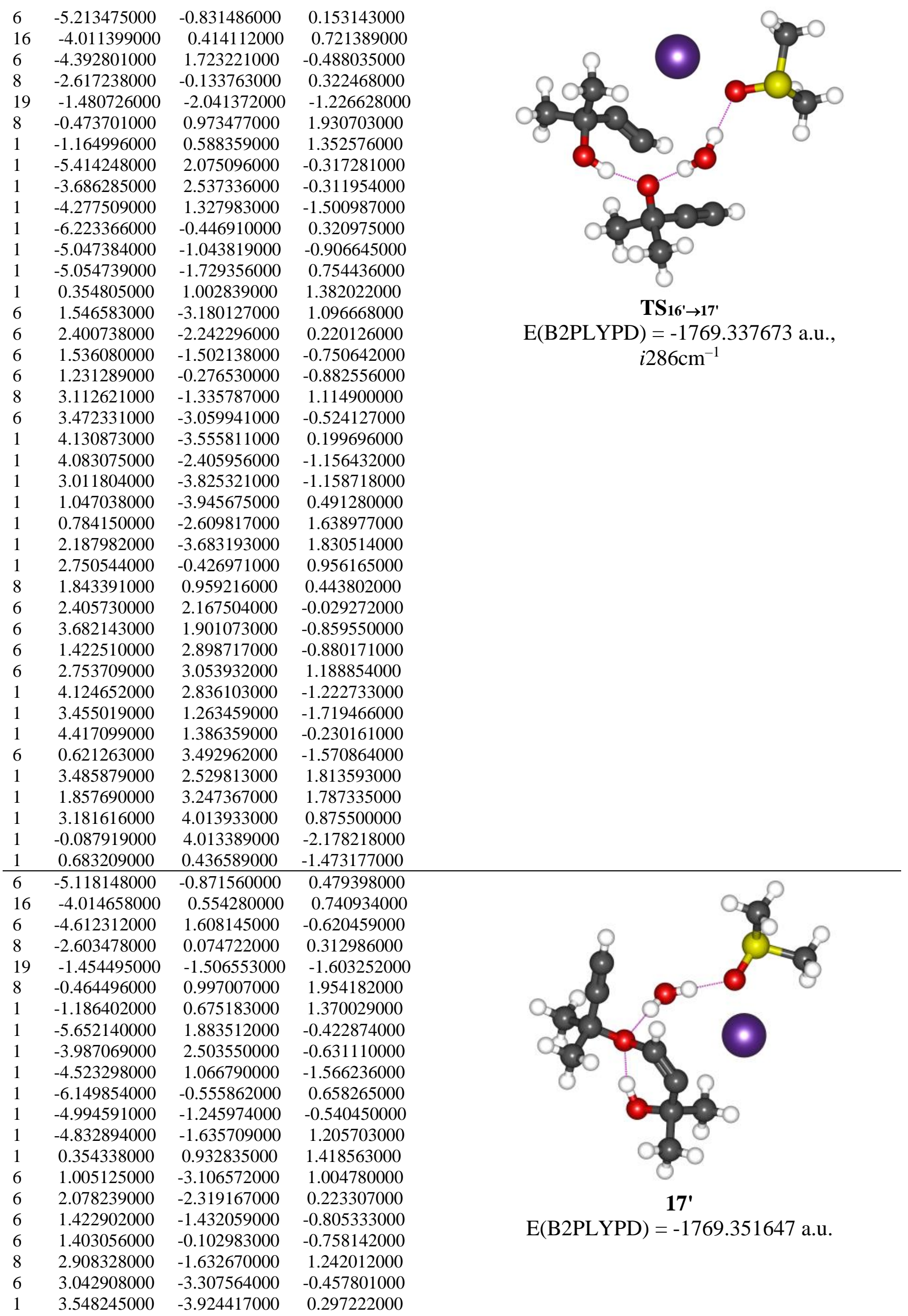




\begin{tabular}{|c|c|c|}
\hline 3.806488000 & -2.764646000 & -1.027529000 \\
\hline 2.507164000 & -3.969885000 & -1.146695000 \\
\hline 0.409915000 & -3.738354000 & 0.335195000 \\
\hline 0.326980000 & -2.419287000 & 1.524621000 \\
\hline 1.482309000 & -3.750940000 & 1.754662000 \\
\hline 2.694210000 & -0.683858000 & 1.159500000 \\
\hline 1.917934000 & 0.733100000 & 0.351614000 \\
\hline 2.658585000 & 1.931414000 & -0.001024000 \\
\hline 3.897659000 & 1.574314000 & -0.840615000 \\
\hline 1.787802000 & 2.869807000 & -0.746402000 \\
\hline 3.069669000 & 2.565004000 & 1.338210000 \\
\hline 4.472205000 & 2.477503000 & -1.070879000 \\
\hline 3.610760000 & 1.093625000 & -1.779810000 \\
\hline 4.534162000 & 0.886090000 & -0.274332000 \\
\hline 1.102640000 & 3.669360000 & -1.345518000 \\
\hline 3.709036000 & 1.867887000 & 1.890088000 \\
\hline 2.187678000 & 2.793068000 & 1.944090000 \\
\hline 3.626623000 & 3.491790000 & 1.166796000 \\
\hline 0.491162000 & 4.367852000 & -1.875820000 \\
\hline 0.939866000 & 0.559180000 & -1.490909000 \\
\hline 4.608570000 & -1.327128000 & -1.412128000 \\
\hline 4.533459000 & 0.008317000 & -0.173083000 \\
\hline 5.092351000 & -0.948807000 & 1.274608000 \\
\hline 3.029761000 & 0.288030000 & 0.057872000 \\
\hline 0.479812000 & -0.467029000 & 0.731793000 \\
\hline 1.773215000 & 2.897011000 & -0.255901000 \\
\hline 2.323898000 & 2.090753000 & -0.188873000 \\
\hline 6.126072000 & -1.266155000 & 1.109530000 \\
\hline 5.042385000 & -0.282947000 & 2.138974000 \\
\hline 4.435653000 & -1.811532000 & 1.416993000 \\
\hline 5.650513000 & -1.636399000 & -1.534455000 \\
\hline 3.987459000 & -2.164613000 & -1.082880000 \\
\hline 4.230696000 & -0.916150000 & -2.350893000 \\
\hline 0.820886000 & 2.552772000 & -0.125600000 \\
\hline-1.937761000 & 2.811094000 & -1.585648000 \\
\hline-1.780185000 & 2.440248000 & -0.078970000 \\
\hline-3.020470000 & 1.623640000 & 0.313188000 \\
\hline-3.244376000 & 0.303947000 & 0.391972000 \\
\hline-0.584611000 & 1.779474000 & 0.146059000 \\
\hline-1.833574000 & 3.762860000 & 0.740970000 \\
\hline-0.984654000 & 4.397937000 & 0.458933000 \\
\hline-1.753347000 & 3.543470000 & 1.813552000 \\
\hline-2.759405000 & 4.332219000 & 0.574807000 \\
\hline-2.886126000 & 3.324241000 & -1.799235000 \\
\hline-1.892733000 & 1.903076000 & -2.199189000 \\
\hline-1.114651000 & 3.472436000 & -1.888025000 \\
\hline-2.314779000 & -0.691852000 & 0.152549000 \\
\hline-2.832107000 & -2.042571000 & -0.064434000 \\
\hline-3.767083000 & -2.077354000 & -1.287679000 \\
\hline-1.625415000 & -2.844592000 & -0.343953000 \\
\hline-3.520424000 & -2.588796000 & 1.202108000 \\
\hline-4.059190000 & -3.109416000 & -1.504408000 \\
\hline-3.261062000 & -1.663218000 & -2.164781000 \\
\hline-4.675024000 & -1.498029000 & -1.095834000 \\
\hline-0.656857000 & -3.533513000 & -0.571535000 \\
\hline-4.446945000 & -2.047803000 & 1.415319000 \\
\hline-2.852605000 & -2.504790000 & 2.064747000 \\
\hline-3.774670000 & -3.643247000 & 1.057133000 \\
\hline 0.197107000 & -4.143299000 & -0.777758000 \\
\hline-4.223703000 & -0.056315000 & 0.695017000 \\
\hline & & \\
\hline
\end{tabular}

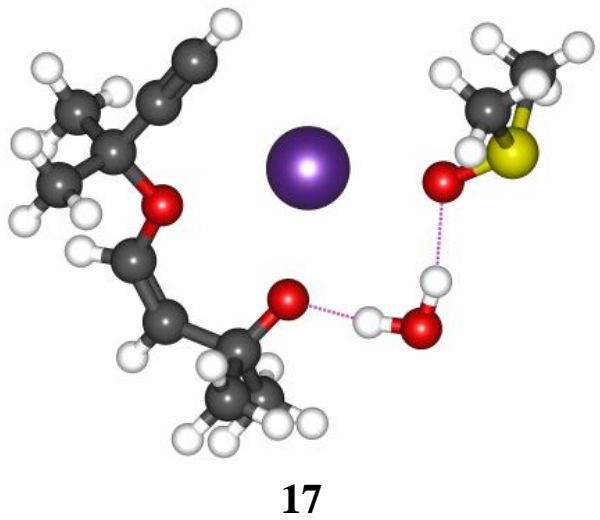

$\mathrm{E}(\mathrm{B} 2 \mathrm{PLYPD})=-1769.411025$ a.u. 


\begin{tabular}{lccc}
8 & -3.543392000 & 0.279726000 & 0.025808000 \\
19 & -1.686149000 & -1.463169000 & 0.151795000 \\
8 & 0.408428000 & 0.268122000 & 0.003745000 \\
16 & 1.916118000 & 0.566030000 & -0.176312000 \\
6 & 2.570729000 & -0.787657000 & -1.207208000 \\
6 & 2.705794000 & 0.101755000 & 1.400159000 \\
1 & -2.486826000 & 1.508992000 & -0.146044000 \\
1 & 3.656864000 & -0.681847000 & -1.281268000 \\
1 & 2.299949000 & -1.748824000 & -0.761412000 \\
1 & 2.120156000 & -0.683711000 & -2.196779000 \\
1 & 3.790640000 & 0.189136000 & 1.291575000 \\
1 & 2.351202000 & 0.805782000 & 2.156264000 \\
1 & 2.418946000 & -0.920103000 & 1.663188000 \\
1 & -0.922141000 & 1.688893000 & -0.188028000 \\
8 & -1.748893000 & 2.208300000 & -0.251337000 \\
1 & -4.438141000 & 0.651633000 & 0.015972000 \\
\hline 6 & 2.929429000 & 1.159295000 & -0.250272000 \\
16 & 1.695170000 & -0.160554000 & -0.500927000 \\
8 & 0.445762000 & 0.269918000 & 0.295446000 \\
6 & 2.506024000 & -1.456589000 & 0.495429000 \\
19 & -2.215022000 & 0.464062000 & 0.461152000 \\
8 & -4.209311000 & -0.591654000 & -0.618151000 \\
1 & 3.459748000 & -1.719930000 & 0.029259000 \\
1 & 2.656344000 & -1.089911000 & 1.514786000 \\
1 & 1.842218000 & -2.324055000 & 0.495897000 \\
1 & 3.874486000 & 0.854390000 & -0.708824000 \\
1 & 2.548631000 & 2.054289000 & -0.747442000 \\
1 & 3.053549000 & 1.341181000 & 0.820922000 \\
1 & -4.976596000 & -1.006618000 & -1.040936000 \\
& & &
\end{tabular}

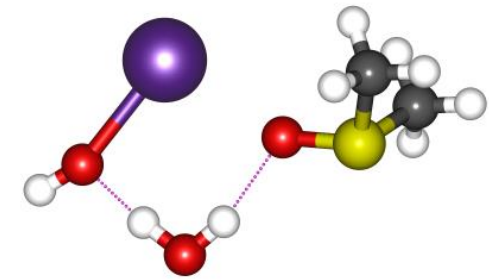

DMSO $\cdot \mathrm{KOH} \cdot \mathrm{H}_{2} \mathrm{O}$

$\mathrm{E}(\mathrm{B} 2 \mathrm{PLYPD})=-1305.062983$ a.u.

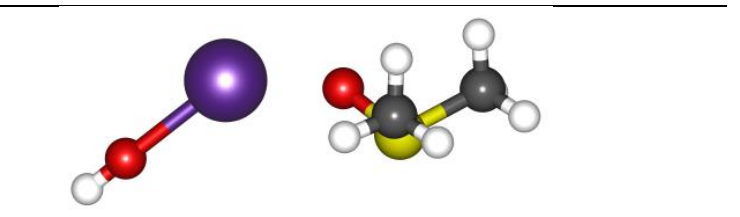

DMSO $\cdot \mathbf{K O H}$

$\mathrm{E}(\mathrm{B} 2 \mathrm{PLYPD})=-1228.647266$ a.u. 